\title{
A!
}

This is an electronic reprint of the original article.

This reprint may differ from the original in pagination and typographic detail.

Tukiainen, Mikko; Lyyra, Henri; Sarbicki, Gniewomir; Maniscalco, Sabrina

\section{Fidelity of dynamical maps}

Published in:

Physical Review A

DOI:

10.1103/PhysRevA.95.052102

Published: 02/05/2017

Document Version

Publisher's PDF, also known as Version of record

Please cite the original version:

Tukiainen, M., Lyyra, H., Sarbicki, G., \& Maniscalco, S. (2017). Fidelity of dynamical maps. Physical Review A, 95(5), 1-14. [052102]. https://doi.org/10.1103/PhysRevA.95.052102

This material is protected by copyright and other intellectual property rights, and duplication or sale of all or part of any of the repository collections is not permitted, except that material may be duplicated by you for your research use or educational purposes in electronic or print form. You must obtain permission for any other use. Electronic or print copies may not be offered, whether for sale or otherwise to anyone who is not an authorised user. 


\title{
Fidelity of dynamical maps
}

\author{
Mikko Tukiainen, ${ }^{1}$ Henri Lyyra, ${ }^{1}$ Gniewomir Sarbicki, ${ }^{2}$ and Sabrina Maniscalco ${ }^{1,3}$ \\ ${ }^{1}$ Turku Centre for Quantum Physics, Department of Physics and Astronomy, University of Turku, \\ FI-20014 Turun yliopisto, Finland \\ ${ }^{2}$ Institute of Physics, Nicolaus Copernicus University, Grudziadzka 5/7, 87-100 Toruń, Poland \\ ${ }^{3}$ Centre for Quantum Engineering, Department of Applied Physics, School of Science, Aalto University, P.O. Box 11000, \\ FIN-00076 Aalto, Finland
}

(Received 12 September 2016; revised manuscript received 15 December 2016; published 2 May 2017)

\begin{abstract}
We introduce the concept of fidelity for dynamical maps in an open quantum system scenario. We derive an inequality linking this quantity to the distinguishability of the inducing environmental states. Our inequality imposes constraints on the allowed set of dynamical maps arising from the microscopic description of system plus environment. Remarkably, the inequality involves only the states of the environment and the dynamical map of the open system and, therefore, does not rely on the knowledge of either the microscopic interaction Hamiltonian or the environmental Hamiltonian characteristic parameters. We demonstrate the power of our result by applying it to two different scenarios: quantum programming and quantum probing. In the first case, we use it to derive bounds on the dimension of the processor for approximate programming of unitaries. In the second case we present an intriguing proof-of-principle demonstration of the ability to extract information on the environment via a quantum probe without any a priori assumption on the form of the system-environment coupling Hamiltonian.
\end{abstract}

DOI: 10.1103/PhysRevA.95.052102

\section{INTRODUCTION}

Quantum systems are extremely sensitive to noise arising from the interaction with their surroundings. This feature is at the very heart of the transition between the quantum and the classical description of the world and, at the same time, imposes limitations on the efficiency of quantum devices for quantum technologies. For this reason, a number of theoretical and experimental approaches focus on the modelization, characterization, and reduction of noise induced by the environment, both by increasing the isolation of the system and by suitable engineering of the environment.

A key quantity in the dynamical description of open quantum systems is the dynamical map, a time-parametrized family of quantum channels [1]. Formally, the exact description of the dynamical map can be obtained starting from a microscopic Hamiltonian model of the system, the environment, and their interaction. In many practical circumstances, however, one does not have accurate knowledge of the microscopic details of either the interaction or the environmental Hamiltonian. In addition, it is well known that different environments and couplings can lead to the same dynamical map. It is therefore clear that, both from an experimental and from a theoretical perspective, it is crucial to develop approaches able to (i) discriminate between different dynamical maps, (ii) identify the restrictions imposed on the form of the dynamical map from the specific choices of the environmental state, Hamiltonian and coupling, and (iii) develop techniques to probe the environment without ideally any a priori assumption on its microscopic properties and on the way it is coupled to the system.

In this paper, we address these issues by introducing a family of channel fidelities, which generalize the familiar (Uhlmann) fidelity of quantum states. Endowed with these quantifiers, we prove a powerful inequality between the fidelities of the two environmental states inducing the dynamics and the corresponding dynamical maps. More precisely, the introduced fidelity quantifies the difference between two dynamical maps by measuring how distinguishable they render a pair of initially identical (or more generally nonorthogonal) states, which are initially uncorrelated from their dynamicsinducing environments. This addresses point (i). the derived inequality, on the other hand, allows us to answer the points (ii) and (iii) since, contrarily to the probing approaches existing in the literature [2-10], it does not rely on the knowledge of either the system-environment interaction Hamiltonian or the characteristic parameters of the environmental Hamiltonian. We prove the usefulness of our inequality by applying it to two different scenarios both highly relevant for the development of quantum technologies, namely quantum programming and quantum probing.

A programmable quantum processor, or gate array, is a device that implements different quantum channels on one system (the data register) depending on the state of another quantum system (the program register). The third element of this device is a fixed array of gates acting on both the data and the program register. The virtue of this arrangement is its versatility: one can realize various maps simply by controlling the state of program register. The alteration of the programming state to perform the desired operation is called quantum programming. Programmable quantum processors were first considered by Nielsen and Chuang in the late 90's [11]. Remarkably, these authors showed that a deterministic universal programmable processor cannot be realised. The reason for this impossibility is that any two states of the program register implementing a pair of inequivalent unitary transformations need to be orthogonal [11-14]. Therefore the programming resources, bounded by the dimension of the program register, are insufficient to implement all unitaries, comprising an uncountable set. 
The no-programming theorem above holds only if the programming is deterministic. Indeed, it was shown already in Ref. [11] that it is possible to successfully implement any onequbit unitary operation with $1 / 4$ probability, thus proving the existence of probabilistic universal quantum processors. This result was generalized in Refs. [15,16], where it was proven that the probability of success is $p=1-\varepsilon$ and the error $\varepsilon$ can be made arbitrarily small; the results were later extend for qudits in Refs. [17,18]. Probabilistic programming, however, tells little about programming states of general quantum transformations, described by completely positive and trace preserving (CPTP) maps. This is, however, important as all realistic implementations of quantum devices are subjected to environmental noise. In this more realistic scenario, known as approximate quantum programming, maps are near to, but not exactly, unitary.

Approximate quantum programming has been studied in Refs. [12,13,15]. In particular, in Refs. [12,13], relations between the overlap of pure programming states and the programmed channels are presented. Our inequality, on the other hand, sets an upper bound for the programming states in terms of the programmed channels and is independent of the choice of the processor. This independence is important since, as we argued above, the microscopic description of the environmental noise affecting the processor may not be known. In addition, unlike in the previously proposed relations, our inequality holds for general (mixed) programming states. In other words, our bound is universally valid and enables us to tackle efficiently problems such as finding size bounds for quantum circuit designs able to implement the desired transformations in full generality.

The second application is in the framework of quantum probing. One particularly renowned quantum feature is summarized by the statement no information without disturbance. Namely, any measurement producing meaningful information generally transforms the state of the measured system. An observer is thus faced with a dilemma: how to ascertain and assign values to properties of the measured system since they may be, and typically are, altered due to the measurement? One way of obtaining some information on the system of interest without predisposing it to a direct measurement is to let it temporarily interact with a smaller ancillary quantum system and then perform a measurement on it: this technique is known as quantum probing. The system of interest in this framework acts as the environment of the quantum probe, and the induced probe dynamics carries information on the properties of the environment. If the coupling between the probe and the system of interest is sufficiently weak, measurements on the probe only lead to small perturbations on the system. In addition, measuring the full probe dynamics ideally allows one to extract even complete information on the system while leading to only minimal disturbance [2-8].

Typical quantum probing strategies assume the knowledge of the microscopic system-probe interaction as well as the system Hamiltonian. The originality of our method is the use of the quantum programming perspective in order to go beyond these existing probing approaches. Namely, by comparing the dynamics induced by an unknown environmental state with the one of a calibration state, some properties on the measured system can be extracted with none or minimal $a$ priori assumptions. Indeed, we provide examples in which such a probing protocol can be used for parameter estimation without knowing anything about the system-probe coupling or the probe Hamiltonian.

The paper is structured as follows. In Sec. II, we introduce the mathematical methods and the notation used throughout this work and provide the main result of this paper: the inequality between the channel fidelity of two state transformations and the fidelity of the corresponding two inducing states. In Sec. III, we present four applications that capture the power of this result from different perspectives. Finally, in Sec. IV, we summarize our results and present conclusions.

\section{INEQUALITY BETWEEN INDUCING STATES AND OPEN SYSTEM DYNAMICS}

We denote a complex separable Hilbert space by $\mathcal{H}$ and the trace class operators on $\mathcal{H}$ by $\mathcal{T}(\mathcal{H})$. A quantum state is represented as a positive operator $\varrho \in \mathcal{T}(\mathcal{H})$ with $\operatorname{Tr}[\varrho]=1$ and the set of quantum states of $\mathcal{H}$ is denoted by $\mathcal{S}(\mathcal{H})$. We indicate with $\operatorname{supp}(\varrho)$ the support of the state $\varrho$ and say that $\varrho_{1}$ and $\varrho_{2}$ are orthogonal, i.e., $\varrho_{1} \perp \varrho_{2}$, when the sets $\operatorname{supp}\left(\varrho_{1}\right)$ and $\operatorname{supp}\left(\varrho_{2}\right)$ are orthogonal. The extremal elements of $\mathcal{S}(\mathcal{H})$ are called pure and such element can be written as $\varrho=|\varphi\rangle\langle\varphi|$ for some unit vector $|\varphi\rangle \in \mathcal{H}$. The transformations of quantum states are represented by linear mappings $\mathcal{E}: \mathcal{T}(\mathcal{H}) \rightarrow \mathcal{T}\left(\mathcal{H}^{\prime}\right)$ that are completely positive and trace-preserving: such transformations are called quantum channels. In particular, a channel $\mathcal{U}$ is called a unitary channel whenever it is of the form $\mathcal{U}(\varrho)=U \varrho U^{\dagger}$ for some unitary operator $U$ on $\mathcal{H}$ and for all $\varrho \in \mathcal{S}(\mathcal{H})$.

The dynamics of a closed quantum system with initial state $\varrho$ is described by a $t$-parametrized group of unitary operators $U^{(t)}$ as $\mathcal{U}^{(t)}(\varrho)=U^{(t)} \varrho U^{(-t)}, t \geqslant 0$. In reality, every physically realizable quantum system $S$, with Hilbert space $\mathcal{H}_{S}$, interacts with some environment, $E$, with Hilbert space $\mathcal{H}_{E}$, such that the pair $S+E$ can be considered a closed system, represented by $\mathcal{H}_{S} \otimes \mathcal{H}_{E}$. One commonly assumes that the system and the environment are initially uncorrelated, that is $\varrho_{S+E}=\varrho \otimes \xi$. The reduced dynamics of the system state can be calculated from the total system dynamics $\mathcal{U}^{(t)}(\varrho \otimes \xi)$ as a partial trace over $\mathcal{H}_{E}$ :

$$
\mathcal{E}^{(t)}(\varrho)=\operatorname{tr}_{E}\left[\mathcal{U}^{(t)}(\varrho \otimes \xi)\right]
$$

In particular, we say that the dynamics $\mathcal{E}^{(t)}(\varrho)$ is induced by the environmental state $\xi$.

The amount of information ascribed in the state $\varrho$ is measured in terms of entropy. In our investigation, we are focusing on a class of $\alpha$-Rényi entropies $S_{\alpha}(\varrho) \doteq \frac{1}{1-\alpha} \ln \operatorname{tr}\left[\varrho^{\alpha}\right]$, $\alpha \in(0,1) \cup(1, \infty)$. The $\alpha$-Rényi entropies generate a oneparameter family of divergences, that from a certain axiomatic point of view for classical random variables $X_{1}$ and $X_{2}$ takes the unique form

$$
D_{\alpha}\left(X_{1} \| X_{2}\right)=\frac{1}{\alpha-1} \sum_{i=1}^{n} \ln \left[p_{1}\left(x_{i}\right)^{\alpha} p_{2}\left(x_{i}\right)^{1-\alpha}\right],
$$

where the two probability measures $p_{1}$ and $p_{2}$ measure the probabilities for outcome $x_{i}, i=1, \ldots, n$, occurring in $X_{1}$ and $X_{2}$, respectively [19]. In a quantum scenario, however, 
the uniqueness of the formulation of the divergences arising from the same set axioms is not guaranteed, and two divergent extensions of Eq. (2) to the quantum setting have been proposed in the literature

$$
\widetilde{S}_{\alpha}\left(\varrho_{1} \| \varrho_{2}\right) \doteq \begin{cases}\frac{1}{\alpha-1} \ln \left\{\operatorname{tr}\left[\varrho_{1}^{\alpha} \varrho_{2}^{1-\alpha}\right]\right\}, & \varrho_{1} \not \perp \varrho_{2} \\ \infty, & \text { otherwise }\end{cases}
$$

and

$$
\begin{aligned}
& S_{\alpha}\left(\varrho_{1} \| \varrho_{2}\right) \\
& \quad \doteq \begin{cases}\frac{1}{\alpha-1} \ln \left[\operatorname{tr}\left[\left(\varrho_{2}^{\frac{1-\alpha}{2 \alpha}} \varrho_{1} \varrho_{2}^{\frac{1-\alpha}{2 \alpha}}\right)^{\alpha}\right]\right], & \text { when } \varrho_{1} \not \perp \varrho_{2} \\
\infty, & \text { otherwise }\end{cases}
\end{aligned}
$$

defined for $\alpha \in(0,1)$ and a pair of quantum states $\varrho_{1}, \varrho_{2} \in$ $\mathcal{S}\left(\mathcal{H}_{S}\right)[20,21]$. In this study, we will be focusing for the latter definition $S_{\alpha}$ and call it the quantum $\alpha$-Rényi divergence. It is, however, worth mentioning that all the results we will present in this paper could be also formulated in the context of $\widetilde{S}_{\alpha}$ and furthermore, since the $S_{\alpha}$ and $\widetilde{S}_{\alpha}$ coincide for commuting states $\left[\varrho_{1}, \varrho_{2}\right]=0$ [20], most of these results are in fact equivalent for the two definitions.

Importantly, many of the most commonly used quantum (relative) entropies, such as the common (relative) entropy, the minimum and the maximum (relative) entropies, can be derived from the quantum $\alpha$-Rényi divergence as special cases; for the properties of $\alpha$-Rényi divergence we refer the reader to Refs. [22-25] and references therein. For our purposes, we recall here the following features:

(S1) $S_{\alpha}\left(\varrho_{1} \| \varrho_{2}\right) \geqslant 0$, where the equality holds if and only if $\varrho_{1}=\varrho_{2}$,

(S2) $S_{\alpha}\left(\varrho_{1} \otimes \xi_{1} \| \varrho_{2} \otimes \xi_{2}\right)=S_{\alpha}\left(\varrho_{1} \| \varrho_{2}\right)+S_{\alpha}\left(\xi_{1} \| \xi_{2}\right)$,

(S3) $S_{\alpha}\left(\mathcal{U}\left(\varrho_{1}\right) \| \mathcal{U}\left(\varrho_{2}\right)\right)=S_{\alpha}\left(\varrho_{1} \| \varrho_{2}\right)$

for all $\alpha \in(0,1)$ and $\varrho_{1}, \varrho_{2} \in \mathcal{S}\left(\mathcal{H}_{S}\right), \xi_{1}, \xi_{2} \in \mathcal{S}\left(\mathcal{H}_{E}\right)$ and for all unitary channels $\mathcal{U}$. Notice that the order of inputs is important, since in general $S_{\alpha}\left(\varrho_{1}, \varrho_{2}\right) \neq S_{\alpha}\left(\varrho_{2}, \varrho_{1}\right)$. Additionally, for all $\alpha \in[1 / 2,1)$, the $\alpha$-Rényi divergence satisfies the data processing inequality

(S4) $S_{\alpha}\left(\mathcal{E}\left(\varrho_{1}\right) \| \mathcal{E}\left(\varrho_{2}\right)\right) \leqslant S_{\alpha}\left(\varrho_{1} \| \varrho_{2}\right)$,

for all $\varrho_{1}, \varrho_{2} \in \mathcal{S}\left(\mathcal{H}_{S}\right)$ and for arbitrary quantum channels $\mathcal{E}$ : $\mathcal{T}\left(\mathcal{H}_{S}\right) \rightarrow \mathcal{T}\left(\mathcal{H}_{S}^{\prime}\right)$

From Eq. (1), it is clear that altering the inducing state $\xi \in \mathcal{S}\left(\mathcal{H}_{E}\right)$, while keeping the coupling interaction fixed, may lead to different channels. A question then arises: how do the two different inducing states and the corresponding induced channels relate to each other? The following proposition provides insight into this question.

Proposition 1. Suppose that two (different) channels $\mathcal{E}_{1}$ and $\mathcal{E}_{2}: \mathcal{T}\left(\mathcal{H}_{S}\right) \rightarrow \mathcal{T}\left(\mathcal{H}_{S}\right)$ are induced by states $\xi_{1}$ and $\xi_{2} \in$ $\mathcal{S}\left(\mathcal{H}_{E}\right)$, respectively, from some fixed (unitary) coupling $\mathcal{U}$ : $\mathcal{T}\left(\mathcal{H}_{S} \otimes \mathcal{H}_{E}\right) \rightarrow \mathcal{T}\left(\mathcal{H}_{S} \otimes \mathcal{H}_{E}\right)$; see Eq. (1). Then for all $\alpha \in$ $[1 / 2,1)$ and $\varrho_{1}, \varrho_{2} \in \mathcal{S}\left(\mathcal{H}_{S}\right)$

$$
S_{\alpha}\left(\mathcal{E}_{1}\left(\varrho_{1}\right) \| \mathcal{E}_{2}\left(\varrho_{2}\right)\right)-S_{\alpha}\left(\varrho_{1} \| \varrho_{2}\right) \leqslant S_{\alpha}\left(\xi_{1} \| \xi_{2}\right) .
$$

Proof. The claim follows from the properties (S1)-(S4), namely,

$$
\begin{aligned}
& S_{\alpha}\left(\varrho_{1} \| \varrho_{2}\right)+S_{\alpha}\left(\xi_{1} \| \xi_{2}\right) \\
& \quad=S_{\alpha}\left(\mathcal{U}\left(\varrho_{1} \otimes \xi_{1}\right) \| \mid \mathcal{U}\left(\varrho_{2} \otimes \xi_{2}\right)\right)
\end{aligned}
$$

$$
\begin{aligned}
& \geqslant S_{\alpha}\left(\operatorname{tr}_{E}\left[\mathcal{U}\left(\varrho_{1} \otimes \xi_{1}\right)\right] \| \operatorname{tr}_{E}\left[\mathcal{U}\left(\varrho_{2} \otimes \xi_{2}\right)\right]\right) \\
& =S_{\alpha}\left(\mathcal{E}_{1}\left(\varrho_{1}\right) \| \mathcal{E}_{2}\left(\varrho_{2}\right)\right) .
\end{aligned}
$$

We will use a short-hand notation

$$
F_{\alpha}\left(\varrho_{1}, \varrho_{2}\right) \doteq \operatorname{tr}\left[\left(\varrho_{2}^{\frac{1-\alpha}{2 \alpha}} \varrho_{1} \varrho_{2}^{\frac{1-\alpha}{2 \alpha}}\right)^{\alpha}\right]
$$

since it will often appear in the following, and call the quantity $F_{\alpha}$ the $\alpha$ fidelity of states. ${ }^{1}$ In the particular case $\alpha=1 / 2, F_{1 / 2}$ corresponds to the usual (Uhlmann) fidelity of states. Using this notation and the monotonicity of the logarithm function, inequality (5) implies

$$
F_{\alpha}\left(\varrho_{1}, \varrho_{2}\right) F_{\alpha}\left(\xi_{1}, \xi_{2}\right) \leqslant F_{\alpha}\left(\mathcal{E}_{1}\left(\varrho_{1}\right), \mathcal{E}_{2}\left(\varrho_{2}\right)\right),
$$

for $\alpha \in[1 / 2,1)$, which can be seen as a generalization of the data processing inequality $F_{\alpha}\left(\varrho_{1}, \varrho_{2}\right) \leqslant F_{\alpha}\left(\mathcal{E}\left(\varrho_{1}\right), \mathcal{E}\left(\varrho_{2}\right)\right)$, for $\alpha \in[1 / 2,1)$. Motivated by this inequality, for $\alpha \in(0,1)$, we define

$$
\mathcal{F}_{\alpha}\left(\mathcal{E}_{1}, \mathcal{E}_{2}\right) \doteq \inf _{\varrho_{1}, \varrho_{2} \in \mathcal{S}\left(\mathcal{H}_{S}\right)} \frac{F_{\alpha}\left(\mathcal{E}_{1}\left(\varrho_{1}\right), \mathcal{E}_{2}\left(\varrho_{2}\right)\right)}{F_{\alpha}\left(\varrho_{1}, \varrho_{2}\right)},
$$

and call it the $\alpha$ fidelity of channels $\mathcal{E}_{1}$ and $\mathcal{E}_{2}$. We justify the terminology, since $\mathcal{F}_{\alpha}$ shares many of the basic properties of fidelity-measures as we shall verify next.

Proposition 2. For all $\alpha \in(0,1)$ and channels $\mathcal{E}_{i}$ : $\mathcal{T}\left(\mathcal{H}_{S}\right) \rightarrow \mathcal{T}\left(\mathcal{H}_{S}\right), \quad i=1$ and $2, \quad \alpha$ fidelity of channels $\mathcal{F}_{\alpha}\left(\mathcal{E}_{1}, \mathcal{E}_{2}\right)$ has the following properties:

(F1) $\mathcal{F}_{\alpha}\left(\mathcal{E}_{1}, \mathcal{E}_{2}\right) \in[0,1]$,

(F2) $\mathcal{F}_{\alpha}\left(\mathcal{E}_{1}, \mathcal{E}_{2}\right)=\mathcal{F}_{\alpha}\left(\mathcal{U} \circ \mathcal{E}_{1} \circ \mathcal{V}, \mathcal{U} \circ \mathcal{E}_{2} \circ \mathcal{V}\right)$, for all unitary channels $\mathcal{U}$ and $\mathcal{V}$.

Additionally, for $\alpha \in[1 / 2,1)$,

(F3) $\mathcal{F}_{\alpha}\left(\mathcal{E}_{1}, \mathcal{E}_{2}\right)=1$ iff $\mathcal{E}_{1}=\mathcal{E}_{2}$,

(F4) $\mathcal{F}_{\alpha}\left(\mathcal{E}_{1}, \mathcal{E}_{2}\right) \leqslant \mathcal{F}_{\alpha}\left(\mathcal{E} \circ \mathcal{E}_{1}, \mathcal{E} \circ \mathcal{E}_{2}\right)$, for all channels $\mathcal{E}$ : $\mathcal{T}\left(\mathcal{H}_{S}\right) \rightarrow \mathcal{T}\left(\mathcal{H}_{S}^{\prime}\right)$

(F5) $\mathcal{F}_{\alpha}\left(\mathcal{E}_{1}, \mathcal{E}_{2}\right) \leqslant \mathcal{F}_{\alpha}\left(\mathcal{E}_{1} \circ \mathcal{E}, \mathcal{E}_{2} \circ \mathcal{E}\right)$, for all channels $\mathcal{E}$ : $\mathcal{T}\left(\mathcal{H}_{S}^{\prime}\right) \rightarrow \mathcal{T}\left(\mathcal{H}_{S}\right)$.

Proof. Clearly $\mathcal{F}_{\alpha}\left(\mathcal{E}_{1}, \mathcal{E}_{2}\right)$ is non-negative by definition. Since $F_{\alpha}\left(\varrho_{1}, \varrho_{2}\right) \leqslant 1$ for all $\varrho_{1}, \varrho_{2} \in \mathcal{S}\left(\mathcal{H}_{S}\right)$, as verified from $(\mathrm{S} 1)$ of $\alpha$-Rényi divergence, $\mathcal{F}_{\alpha}\left(\mathcal{E}_{1}, \mathcal{E}_{2}\right) \leqslant$ $\inf _{\varrho_{1}, \varrho_{2}} F_{\alpha}\left(\varrho_{1}, \varrho_{2}\right)^{-1}=1$ proving $(\mathrm{F} 1)$. Using the properties (S3) and (S4) of $\alpha$-Rényi divergence, the monotonicity of the logarithm function and the fact $\mathcal{S}\left(\mathcal{H}_{S}\right) \simeq \mathcal{U}\left(\mathcal{S}\left(\mathcal{H}_{S}\right)\right)$ we conclude $(\mathrm{F} 2)$ and $(\mathrm{F} 4)$. Since $F_{\alpha}\left(\varrho_{1}, \varrho_{2}\right) \leqslant F_{\alpha}\left(\mathcal{E}\left(\varrho_{1}\right), \mathcal{E}\left(\varrho_{2}\right)\right)$, for all channels $\mathcal{E}$ when $\alpha \in[1 / 2,1)$, we confirm $\mathcal{F}_{\alpha}(\mathcal{E}, \mathcal{E})=$ 1. Assume, on the other hand, that $\mathcal{F}_{\alpha}\left(\mathcal{E}_{1}, \mathcal{E}_{2}\right)=1$, but make a counterassumption $\mathcal{E}_{1} \neq \mathcal{E}_{2}$. Then, there exists a state $\varrho \in \mathcal{S}\left(\mathcal{H}_{S}\right)$ such that $\mathcal{E}_{1}(\varrho) \neq \mathcal{E}_{2}(\varrho)$ and therefore by $(\mathrm{S} 1) F_{\alpha}\left(\mathcal{E}_{1}(\varrho), \mathcal{E}_{2}(\varrho)\right)<1$. Since $1=\mathcal{F}_{\alpha}\left(\mathcal{E}_{1}, \mathcal{E}_{2}\right) \leqslant$ $F_{\alpha}\left(\mathcal{E}_{1}(\varrho), \mathcal{E}_{2}(\varrho)\right)<1$, this leads to a contradiction and we get (F3). Lastly, we prove (F5). From the properties above, we get the inequality

$$
\begin{aligned}
& \frac{F_{\alpha}\left(\left(\mathcal{E}_{1} \circ \mathcal{E}\right)\left(\varrho_{1}\right),\left(\mathcal{E}_{2} \circ \mathcal{E}\right)\left(\varrho_{2}\right)\right)}{F_{\alpha}\left(\mathcal{E}\left(\varrho_{1}\right), \mathcal{E}\left(\varrho_{2}\right)\right)} \\
& \leqslant \frac{F_{\alpha}\left(\left(\mathcal{E}_{1} \circ \mathcal{E}\right)\left(\varrho_{1}\right),\left(\mathcal{E}_{2} \circ \mathcal{E}\right)\left(\varrho_{2}\right)\right)}{F_{\alpha}\left(\varrho_{1}, \varrho_{2}\right)},
\end{aligned}
$$

\footnotetext{
${ }^{1}$ In one of the founding papers [21], this quantity goes by the name "sandwiched quasirelative entropy."
} 
which together with $\mathcal{E}\left(\mathcal{S}\left(\mathcal{H}_{S}^{\prime}\right)\right) \subset \mathcal{S}\left(\mathcal{H}_{S}\right)$ implies that

$$
\begin{aligned}
\mathcal{F}_{\alpha}\left(\mathcal{E}_{1}, \mathcal{E}_{2}\right) & \leqslant \inf _{\mathcal{E}\left(\varrho_{1}\right), \mathcal{E}\left(\varrho_{2}\right)} \frac{F_{\alpha}\left(\mathcal{E}_{1}\left(\mathcal{E}\left(\varrho_{1}\right)\right), \mathcal{E}_{2}\left(\mathcal{E}\left(\varrho_{2}\right)\right)\right)}{F_{\alpha}\left(\mathcal{E}\left(\varrho_{1}\right), \mathcal{E}\left(\varrho_{2}\right)\right)} \\
& =\inf _{\varrho_{1}, \varrho_{2}} \frac{F_{\alpha}\left(\left(\mathcal{E}_{1} \circ \mathcal{E}\right)\left(\varrho_{1}\right),\left(\mathcal{E}_{2} \circ \mathcal{E}\right)\left(\varrho_{2}\right)\right)}{F_{\alpha}\left(\mathcal{E}\left(\varrho_{1}\right), \mathcal{E}\left(\varrho_{2}\right)\right)} \\
& \leqslant \inf _{\varrho_{1}, \varrho_{2}} \frac{F_{\alpha}\left(\left(\mathcal{E}_{1} \circ \mathcal{E}\right)\left(\varrho_{1}\right),\left(\mathcal{E}_{2} \circ \mathcal{E}\right)\left(\varrho_{2}\right)\right)}{F_{\alpha}\left(\varrho_{1}, \varrho_{2}\right)} \\
& =\mathcal{F}_{\alpha}\left(\mathcal{E}_{1} \circ \mathcal{E}, \mathcal{E}_{2} \circ \mathcal{E}\right) .
\end{aligned}
$$

Different fidelity measures of pairs of quantum channels have been formulated including the process fidelity measuring the distinguishability of the corresponding Choi states [26], the minimax fidelity [27] with operational connection to the single-shot discrimination of channels done with the so-called process POVMs [28,29] and the plethora of gate fidelities considered in Refs. [30,31]. All of these channel fidelities have a common feature: they measure the distinguishability of pair of quantum channels in different operational scenarios. The $\alpha$ fidelity of channels we propose does not, however, admit operational connection to the above channel discrimination tasks due to a peculiar property the other channel fidelities do not share. Namely, as we will see shortly, it vanishes for any pair of different unitary channels. This unique feature makes our fidelity particularly appealing in quantum programming.

We note that the double infima in Eq. (9) can make $\mathcal{F}_{\alpha}$ difficult to evaluate. It is, however, readily verified that $\mathcal{F}_{\alpha}\left(\mathcal{E}_{1}, \mathcal{E}_{2}\right) \leqslant \inf _{\varrho} F_{\alpha}\left(\mathcal{E}_{1}(\varrho), \mathcal{E}_{2}(\varrho)\right)$, where the latter quantity also sets an upper bound for the inducing states and it is much easier to handle. Furthermore, since it has been shown in [32] that $F_{\alpha}$ is jointly concave for $\alpha \in[1 / 2,1)$, that is, in particular for an arbitrary $\varrho=$ $\sum_{i} \lambda_{i}|i\rangle\langle i|$ it satisfies $F_{\alpha}\left(\mathcal{E}_{1}\left(\sum_{i} \lambda_{i}|i\rangle\langle i|\right), \mathcal{E}_{2}\left(\sum_{i} \lambda_{i}|i\rangle\langle i|\right)\right) \geqslant$ $\sum_{i} \lambda_{i} F_{\alpha}\left(\mathcal{E}_{1}(|i\rangle\langle i|), \mathcal{E}_{2}(|i\rangle\langle i|)\right)$ for $\alpha \in[1 / 2,1)$, it can be concluded that it is enough to consider the infimum over the set of pure states. In the special case $\alpha=1 / 2$, the quantity $F_{\min }\left(\mathcal{E}_{1}, \mathcal{E}_{2}\right) \doteq \inf _{\varrho} F_{1 / 2}\left(\mathcal{E}_{1}(\varrho), \mathcal{E}_{2}(\varrho)\right)$ is known as the minimal gate fidelity $[30,31]$. We leave it as an open problem, whether also in $\mathcal{F}_{\alpha}$ it is sufficient to evaluate the infima over the set pure states or not.

Above an ordering, $\mathcal{F}_{1 / 2} \leqslant F_{\min }$, was pointed out, and one may wish to reveal such relations between other fidelities also, e.g., between $\alpha$ fidelity and the commonly used process fidelity defined for channels $\mathcal{E}_{i}: \mathcal{T}\left(\mathcal{H}_{S}\right) \rightarrow$ $\mathcal{T}\left(\mathcal{H}_{S}\right), \quad i=1,2, \quad$ via $\quad F_{\text {proc }}\left(\mathcal{E}_{1}, \mathcal{E}_{2}\right) \doteq F_{1 / 2}\left(\mathcal{E}_{1} \otimes \mathcal{I}(\Omega), \mathcal{E}_{2} \otimes\right.$ $\mathcal{I}(\Omega)$ ), where $\mathcal{I}: \mathcal{T}\left(\mathcal{H}_{S}\right) \rightarrow \mathcal{T}\left(\mathcal{H}_{S}\right)$ is the identity channel and $\Omega=\frac{1}{\operatorname{dim}\left(\mathcal{H}_{S}\right)} \sum_{n, m=1}^{\operatorname{dim}\left(\mathcal{H}_{S}\right)}|n n\rangle\langle m m|$ is the maximally entangled state in $\mathcal{H}_{S} \otimes \mathcal{H}_{S}$ with respect to an orthonormal basis $\left\{|n\rangle, n=1, \ldots, \operatorname{dim}\left(\mathcal{H}_{S}\right)\right\}$. Such an ordering cannot, however, be established. Namely, it can be confirmed that for the onequbit channels $\mathcal{E}_{1}=\mathcal{I}$ and $\mathcal{E}_{2}(\varrho)=1 / 3 \sum_{i=1}^{3} \sigma_{i} \varrho \sigma_{i}^{\dagger}$, where $\sigma_{i}, i=1,2,3$, are the Pauli matrices, the process fidelity vanishes, $F_{\text {proc }}\left(\mathcal{E}_{1}, \mathcal{E}_{2}\right)=0$, or more generally $F_{\alpha}\left(\mathcal{E}_{1} \otimes \mathcal{I}, \mathcal{E}_{2} \otimes\right.$ $\mathcal{I}(\Omega))=0$ for all $\alpha \in(0,1)$. On the other hand, by writing $\varrho_{\vec{m}}=\frac{1}{2}(\mathbb{1}+\vec{m} \cdot \vec{\sigma})$, where $\vec{m} \cdot \vec{\sigma}=\sum_{i=1}^{3} m_{i} \sigma_{i}$ and $\vec{m} \in \mathbb{R}^{3}$ has $\|\vec{m}\| \leqslant 1$, we see that $\mathcal{E}_{2}\left(\varrho_{\vec{m}}\right)=\frac{2}{3} \cdot\left(\frac{1}{2} \mathbb{1}\right)+\frac{1}{3} \cdot \varrho_{-\vec{m}}$ and therefore

$$
\begin{aligned}
\mathcal{F}_{\alpha}\left(\mathcal{E}_{1}, \mathcal{E}_{2}\right) & =\inf _{\varrho_{\vec{n}}, \varrho_{\vec{m}}} \frac{F_{\alpha}\left(\varrho_{\vec{n}}, \frac{2}{3} \cdot\left(\frac{1}{2} \mathbb{1}\right)+\frac{1}{3} \cdot \varrho_{-\vec{m}}\right)}{F_{\alpha}\left(\varrho_{\vec{n}}, \varrho_{\vec{m}}\right)} \\
& \geqslant \frac{2}{3} \inf _{\varrho_{\vec{n}}} F_{\alpha}\left(\varrho_{\vec{n}}, 1 / 2 \mathbb{1}\right)+\frac{1}{3} \inf _{\varrho_{\vec{n}}, \varrho_{\vec{m}}} \frac{F_{\alpha}\left(\varrho_{\vec{n}}, \varrho_{-\vec{m}}\right)}{F_{\alpha}\left(\varrho_{\vec{n}}, \varrho_{\vec{m}}\right)} \\
& =\frac{2}{3}\left(\frac{1}{2}\right)^{1-\alpha} \neq 0,
\end{aligned}
$$

for $\alpha \in[1 / 2,1)$, where the estimation follows from the joint concavity of the $\alpha$ fidelity [32]. Therefore there exist channels $\mathcal{E}_{1}$ and $\mathcal{E}_{2}$ for which $F_{\text {proc }}\left(\mathcal{E}_{1}, \mathcal{E}_{2}\right)<$ $\mathcal{F}_{\alpha}\left(\mathcal{E}_{1}, \mathcal{E}_{2}\right)$ for all $\alpha \in[1 / 2,1)$. However, in Proposition III A, we will show that $\mathcal{F}_{\alpha}\left(\mathcal{U}_{1}, \mathcal{U}_{2}\right)=0$ for any pair of unitary channels $\mathcal{U}_{1} \neq \mathcal{U}_{2}$, which does not hold for $F_{\text {proc }}$ in general [26]. In conclusion, there is no ordering between $\mathcal{F}_{\alpha}$ and $F_{\text {proc }}$. Furthermore, since the process fidelity and the minimax fidelity [27] $F_{\text {mm }}\left(\mathcal{E}_{1}, \mathcal{E}_{2}\right) \doteq$ $\inf _{\varrho \in \mathcal{S}\left(\mathcal{H}_{S}\right)} \operatorname{tr}\left[\left|\sqrt{\mathcal{E}_{1} \otimes \mathcal{I}(\Omega)} \mathbb{1} \otimes \varrho \sqrt{\mathcal{E}_{2} \otimes \mathcal{I}(\Omega)}\right|\right]$ satisfy $F_{\mathrm{mm}} \leqslant$ $F_{\text {proc }}$, and since there exist unequal unitary channels $\mathcal{U}_{i}$, $i=1$ and 2 , such that $F_{\mathrm{mm}}\left(\mathcal{U}_{1}, \mathcal{U}_{2}\right) \neq 0$, it may be concluded that the channel fidelities $F_{\mathrm{mm}}$ and $\mathcal{F}_{\alpha}$ are not related either. As a corollary, we can prove that the $\alpha$ fidelity of channels is not (sub)multiplicative for separable channels.

Proposition 3. $\mathcal{F}_{\alpha}$ does not satisfy the multiplicativity property

$$
\mathcal{F}_{\alpha}\left(\mathcal{E}_{1}, \mathcal{E}_{2}\right) \mathcal{F}_{\alpha}\left(\mathcal{C}_{1}, \mathcal{C}_{2}\right)=\mathcal{F}_{\alpha}\left(\mathcal{E}_{1} \otimes \mathcal{C}_{1}, \mathcal{E}_{2} \otimes \mathcal{C}_{2}\right)
$$

for all channels $\mathcal{E}_{i}: \mathcal{T}\left(\mathcal{H}_{S}\right) \rightarrow \mathcal{T}\left(\mathcal{H}_{S}\right), \mathcal{C}_{i}: \mathcal{T}\left(\mathcal{H}_{S}^{\prime}\right) \rightarrow \mathcal{T}\left(\mathcal{H}_{S}^{\prime}\right)$, $i=1$ and 2 .

Proof. It is easily seen that $\mathcal{F}_{\alpha}\left(\mathcal{E}_{1}, \mathcal{E}_{2}\right) \mathcal{F}_{\alpha}\left(\mathcal{C}_{1}, \mathcal{C}_{2}\right) \geqslant \mathcal{F}_{\alpha}\left(\mathcal{E}_{1} \otimes\right.$ $\left.\mathcal{C}_{1}, \mathcal{E}_{2} \otimes \mathcal{C}_{2}\right)$, since $\mathcal{S}\left(\mathcal{H}_{S}\right) \otimes \mathcal{S}\left(\mathcal{H}_{S}^{\prime}\right) \subset \mathcal{S}\left(\mathcal{H}_{S} \otimes \mathcal{H}_{S}^{\prime}\right)$. The other direction, however, leads to a contradiction. Namely, assume that $\mathcal{F}_{\alpha}\left(\mathcal{E}_{1}, \mathcal{E}_{2}\right) \mathcal{F}_{\alpha}\left(\mathcal{C}_{1}, \mathcal{C}_{2}\right) \leqslant \mathcal{F}_{\alpha}\left(\mathcal{E}_{1} \otimes \mathcal{C}_{1}, \mathcal{E}_{2} \otimes \mathcal{C}_{2}\right)$ holds for all channels $\mathcal{E}_{i}: \mathcal{T}\left(\mathcal{H}_{S}\right) \rightarrow \mathcal{T}\left(\mathcal{H}_{S}\right), \mathcal{C}_{i}: \mathcal{T}\left(\mathcal{H}_{S}^{\prime}\right) \rightarrow \mathcal{T}\left(\mathcal{H}_{S}^{\prime}\right), i=$ 1 and 2 . Then in particular

$$
\begin{aligned}
\mathcal{F}_{\alpha}\left(\mathcal{E}_{1}, \mathcal{E}_{2}\right) & \leqslant \mathcal{F}_{1 / 2}\left(\mathcal{E}_{1} \otimes \mathcal{I}, \mathcal{E}_{2} \otimes \mathcal{I}\right) \\
& \leqslant F_{\alpha}\left(\mathcal{E}_{1} \otimes \mathcal{I}(\Omega), \mathcal{E}_{2} \otimes \mathcal{I}(\Omega)\right)
\end{aligned}
$$

which we have noted before does not hold in general.

We observe that similar calculations to those above can be used to verify that $F_{\min }$ is not multiplicative either. So far, we have only considered static quantum channels, however, the calculations above hold as well for dynamical maps. The emerging inequality

$$
F_{\alpha}\left(\xi_{1}, \xi_{2}\right) \leqslant \mathcal{F}_{\alpha}\left(\mathcal{E}_{1}^{(t)}, \quad \mathcal{E}_{2}^{(t)}\right), \quad \alpha \in[1 / 2,1), \quad t \geqslant 0,
$$

has the following twofold interpretation. Firstly, if the initial environmental states $\xi_{i}, i=1$ and 2 , induce dynamics $\mathcal{E}_{i}^{(t)}$, respectively, then $\mathcal{F}_{\alpha}\left(\mathcal{E}_{1}^{(t)}, \mathcal{E}_{2}^{(t)}\right)$ cannot decrease below $F_{\alpha}\left(\xi_{1}, \xi_{2}\right)$ for any $t \geqslant 0$. This sets limitations on what dynamics are compatible with the inducing environmental states. On the other hand, since $\inf _{t \geqslant 0} \mathcal{F}_{\alpha}\left(\mathcal{E}_{1}^{(t)}, \mathcal{E}_{2}^{(t)}\right)$ majorizes $F_{\alpha}\left(\xi_{1}, \xi_{2}\right)$, it is possible to gain some information about the environment by measuring the open system dynamics, i.e., by probing. Remarkably, these two strategies work even if the environment and the interaction were not specified. The next section is devoted to examples that demonstrate these properties. 
(a)

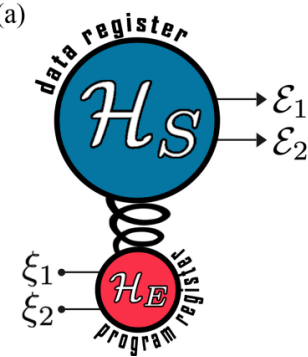

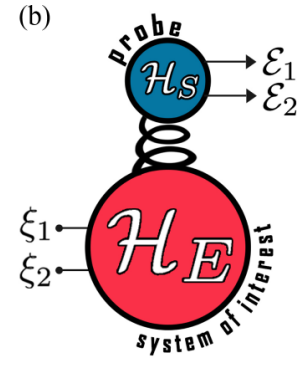

FIG. 1. Quantum programming and probing. (a) In a quantum programming scheme, one varies the state $\xi_{i}$ of the program register $\left(\mathcal{H}_{E}\right)$ coupled to the data register $\left(\mathcal{H}_{S}\right)$. By varying $\xi_{i}$ we can induce multiple different channels $\mathcal{E}_{i}$ on the data register. In this scenario, the emphasis is on $\mathcal{H}_{S}$. (b) In a quantum probing scheme, on the other hand, the system of interest is $\mathcal{H}_{E}$, which is coupled to the probe $\mathcal{H}_{S}$. Different initial states, $\xi_{i}$, of the system lead to different dynamics, $\mathcal{E}_{i}$, of the probe. By measuring the probe dynamics $\mathcal{E}_{i}$, we can obtain some information of the corresponding inducing state $\xi_{i}$.

\section{APPLICATIONS OF THE INEQUALITY}

In the following, we will present three applications of inequality (8) for quantum programming and quantum probing purposes. Figure 1 illustrates the quantum probing and quantum programming protocols. Despite their apparent similarity, the main objectives in the two protocols are complementary; in quantum programming, the aim is to induce different channels for the data register in a controlled way, whereas in quantum probing the goal is to extract information of the system of interest which induced the dynamics.

\section{A. Quantum programming}

The altering of the inducing state $\xi \in \mathcal{S}\left(\mathcal{H}_{E}\right)$ in Eq. (1) is an action known as quantum programming and accordingly, the pair $\left\langle\mathcal{H}_{E}, \mathcal{U}\right\rangle$ formed by the program space $\mathcal{H}_{E}$ and the (unitary) coupling $\mathcal{U}$ between $\mathcal{H}_{S}$ and $\mathcal{H}_{E}$ is called a programmable processor.

From the inequality

$$
F_{\alpha}\left(\xi_{1}, \xi_{2}\right) \leqslant \mathcal{F}_{\alpha}\left(\mathcal{E}_{1}, \mathcal{E}_{2}\right), \quad \alpha \in[1 / 2,1),
$$

we get a limit for the fidelity of the inducing states in terms of the induced channels. We recall once again that a sharp equality between the inducing states and the induced channels has been introduced in Ref. [33]. This equality depends on the programmable processor. In inequality (16), on the contrary, no such dependence is present.

In the case $\alpha=1 / 2$, inequality (16) has a particularly nice interpretation, viz. $F_{1 / 2}\left(\xi_{1}, \xi_{2}\right)$ measures the distinguishability of the states $\xi_{i}, i=1$ and 2 . Also, $\mathcal{F}_{1 / 2}$ has a similar interpretation: $\mathcal{F}_{1 / 2}\left(\mathcal{E}_{1}, \mathcal{E}_{2}\right)=\inf _{\varrho_{1}, \varrho_{2}} \frac{F_{1 / 2}\left(\mathcal{E}_{1}\left(\varrho_{1}\right), \mathcal{E}_{2}\left(\varrho_{2}\right)\right)}{F_{1 / 2}\left(\varrho_{1}, \varrho_{2}\right)}$ quantifies the mutual distinguishability of the channels $\mathcal{E}_{1}, \mathcal{E}_{2}$ by measuring how distinguishable these channels can render a pair of initially nonorthogonal states $\varrho_{1}$ and $\varrho_{2}$. The inequality then naturally stems from the fact that the distinguishabilities should not increase under physical processes, such as programming. We connect inequality (16) to earlier known programming results [11-14] by proving that unitary channels are, in this sense, the most distinguishable.

Proposition 4. Let $\mathcal{U}_{1}$ and $\mathcal{U}_{2}$ be two unitary channels. If $\mathcal{U}_{1} \neq \mathcal{U}_{2}$ then $\mathcal{F}_{\alpha}\left(\mathcal{U}_{1}, \mathcal{U}_{2}\right)=0$ for all $\alpha \in(0,1)$. In particular, the inducing states of different unitary channels must be orthogonal regardless of the choice of the processor $\left\langle\mathcal{H}_{E}, \mathcal{U}\right\rangle$.

Proof. We first prove the proposition for $\alpha=1 / 2$. Using the properties of fidelity of pure states, we have $F_{1 / 2}\left(\mathcal{U}_{1}\left(\left|\varphi_{1}\right\rangle\left\langle\varphi_{1}\right|\right), \mathcal{U}_{2}\left(\left|\varphi_{2}\right\rangle\left\langle\varphi_{2}\right|\right)\right)=\left|\left\langle\varphi_{1} \mid U_{1}^{\dagger} U_{2} \varphi_{2}\right\rangle\right|$. It suffices to show that for any operator $U \neq c \cdot \mathbb{1}_{\mathcal{H}_{S}},|c|=1$, there exists nonorthogonal unit vectors $\left|\varphi_{1}\right\rangle,\left|\varphi_{2}\right\rangle \in \mathcal{H}_{S}$ such that $\left\langle\varphi_{1} \mid U \varphi_{2}\right\rangle=0$. Make a counterassumption that $\left\langle\varphi_{1} \mid U \varphi_{2}\right\rangle \neq$ 0 whenever $\left\langle\varphi_{1} \mid \varphi_{2}\right\rangle \neq 0$. One can express $\left|\varphi_{2}\right\rangle=\alpha\left|\varphi_{1}\right\rangle+$ $\beta|\eta\rangle$, where $\left\langle\varphi_{1} \mid \eta\right\rangle=0$ and $|\alpha|^{2}+|\beta|^{2}=1$. Fix $\left|\varphi_{1}\right\rangle$ such that $\left|\left\langle\varphi_{1} \mid U \varphi_{1}\right\rangle\right| \neq 1$. We can then choose a unit vector $|\eta\rangle=\left(U^{\dagger} \varphi_{1}-\left\langle\varphi_{1} \mid U^{\dagger} \varphi_{1}\right\rangle \varphi_{1}\right) / \sqrt{1-\left|\left\langle\varphi_{1} \mid U \varphi_{1}\right\rangle\right|^{2}}$ orthogonal to $\varphi_{1}$. With the above choices, we have $0 \neq\left\langle\varphi_{1} \mid U \varphi_{2}\right\rangle=$ $\alpha\left\langle\varphi_{1} \mid U \varphi_{1}\right\rangle+\beta \sqrt{1-\left|\left\langle\varphi_{1} \mid U \varphi_{1}\right\rangle\right|^{2}}$ for all $\alpha$ and $\beta$. Since $\alpha=$ $\sqrt{1-\left|\left\langle\varphi_{1} \mid U \varphi_{1}\right\rangle\right|^{2}}$ and $\beta=-\left\langle\varphi_{1} \mid U \varphi_{1}\right\rangle$ contradict this while satisfying $|\alpha|^{2}+|\beta|^{2}=1$, the counterassumption is falsified. Going the other way around, for any unit vector $\left|\varphi_{1}\right\rangle$ such that $\left|\left\langle\varphi_{1} \mid U \varphi_{1}\right\rangle\right| \neq 1$, we find that the unit vector

$$
\begin{aligned}
\left|\varphi_{2}\right\rangle= & \sqrt{1-\left|\left\langle\varphi_{1} \mid U \varphi_{1}\right\rangle\right|^{2}}\left|\varphi_{1}\right\rangle \\
& -\frac{\left\langle\varphi_{1} \mid U \varphi_{1}\right\rangle}{\sqrt{1-\left|\left\langle\varphi_{1} \mid U \varphi_{1}\right\rangle\right|^{2}}}\left(U^{\dagger}\left|\varphi_{1}\right\rangle-\left\langle\varphi_{1} \mid U^{\dagger} \varphi_{1}\right\rangle\left|\varphi_{1}\right\rangle\right),
\end{aligned}
$$

which satisfies $\left\langle\varphi_{1} \mid U \varphi_{2}\right\rangle=0$ and $\left\langle\varphi_{1} \mid \varphi_{2}\right\rangle \neq 0$. Fixing $U=$ $U_{1}^{\dagger} U_{2}$ proves that $\mathcal{F}_{1 / 2}\left(\mathcal{U}_{1}, \mathcal{U}_{2}\right)=0$. For general $\alpha \in(0,1)$ the claim follows from the above considerations when noticing that $F_{\alpha}\left(\varrho_{1}, \varrho_{2}\right)=0$ if and only if $\varrho_{1} \perp \varrho_{2}$; see Eq. (4).

It should be stressed that for any pair of quantum channels $\mathcal{E}_{i}, i=1$ and 2 acting on a same Hilbert space, one can find a processor $\left\langle\mathcal{H}_{E}, \mathcal{U}\right\rangle$ from which the two channels can be induced. To see this, let us recall that any channel $\mathcal{E}: \mathcal{T}\left(\mathcal{H}_{S}\right) \rightarrow \mathcal{T}\left(\mathcal{H}_{S}\right)$ admits a Stinespring dilation of the form $\mathcal{E}(\varrho)=\operatorname{tr}_{\mathcal{H}_{S}}\left[G \varrho \otimes|\eta\rangle\langle\eta| G^{\dagger}\right]$, where $G$ is a unitary on $\mathcal{H}_{S} \otimes \mathcal{H}_{S}$ and $|\eta\rangle \in \mathcal{H}_{S}$ is some fixed unit vector. We can therefore assume without loss of generality that $\mathcal{E}_{i}(\varrho)=$ $\operatorname{tr}_{\mathcal{H}_{S}}\left[G_{i} \varrho \otimes|\eta\rangle\langle\eta| G_{i}^{\dagger}\right]$ for unitaries $G_{i}, i=1$ and 2 . Consider any pair of orthogonal unit vectors $\left|\phi_{1}\right\rangle$ and $\left|\phi_{2}\right\rangle,\left\langle\phi_{1} \mid \phi_{2}\right\rangle=0$ in a two-dimensional Hilbert space $\mathbb{C}^{2}$. Then, it is easily verified that $U=G_{1} \otimes|\eta\rangle\left\langle\eta|\otimes| \phi_{1}\right\rangle\left\langle\phi_{1}\left|+G_{2} \otimes\right| \eta\right\rangle\langle\eta| \otimes$ $\left|\phi_{2}\right\rangle\left\langle\phi_{2}\right|+\mathbb{1}_{\mathcal{H}_{S}} \otimes\left(\mathbb{1}_{\mathcal{H}_{S}}-|\eta\rangle\langle\eta|\right) \otimes \mathbb{1}_{\mathbb{C}^{2}}$ defines a unitary operator on $\mathcal{H}_{S} \otimes\left(\mathcal{H}_{S} \otimes \mathbb{C}^{2}\right)$. It follows that, by defining $\mathcal{H}_{E}=$ $\mathcal{H}_{S} \otimes \mathbb{C}^{2}$ and $\mathcal{U}(\varrho)=U \varrho U^{\dagger}$, the vectors $\left|\eta \otimes \phi_{i}\right\rangle$ program the two channels $\mathcal{E}_{i}, i=1$ and 2 , via processor $\left\langle\mathcal{H}_{E}, \mathcal{U}\right\rangle$. As a result, any pair of quantum channels, even identical ones, can be programmed with orthogonal programming states. On the other hand, we showed above that for identical quantum channels the channel $\alpha$ fidelity reaches its maximum value 1. Importantly, this example alludes that is impossible to generally talk about the tightness of inequality (16).

In connection to the above, there are two complementary questions related to the tightness of inequality (16) that we would like to address. Firstly, one may wonder if for any pair of programming states $\xi_{i} \in \mathcal{S}\left(\mathcal{H}_{E}\right)$ there exists a processor $\left\langle\mathcal{H}_{E}, \mathcal{U}\right\rangle$ realizing channels $\mathcal{E}_{i}, i=1$ and 2, respectively, that would satisfy $F_{\alpha}\left(\xi_{1}, \xi_{2}\right)=\mathcal{F}_{\alpha}\left(\mathcal{E}_{1}, \mathcal{E}_{2}\right)$. The answer is affirmative, namely any quantum state $\xi \in \mathcal{S}\left(\mathcal{H}_{E}\right)$ can be considered 
as the preparation channel $\mathcal{E}_{\xi}: \mathcal{T}\left(\mathcal{H}_{E}\right) \rightarrow \mathbb{C}\{\xi\}, \mathcal{E}_{\xi}(\varrho)=\xi$, for all $\varrho \in \mathcal{S}\left(\mathcal{H}_{E}\right)$. Moreover, any such channel may be programmed with state $\xi \in \mathcal{S}\left(\mathcal{H}_{E}\right)$ by using the processor $\left\langle\mathcal{H}_{E}, \mathcal{U}_{\mathrm{SWAP}}\right\rangle$, where $\mathcal{U}_{\mathrm{SWAP}}\left(T_{1} \otimes T_{2}\right)=T_{2} \otimes T_{1}$, for all $T_{i} \in$ $\mathcal{T}\left(\mathcal{H}_{E}\right)$, that is, $\mathcal{E}_{\xi}(\varrho)=\operatorname{tr}_{\mathcal{H}_{E}}\left[\mathcal{U}_{\text {SWAP }}(\varrho \otimes \xi)\right]$, for all $\varrho \in$ $\mathcal{S}\left(\mathcal{H}_{E}\right)$. It is evident that in this case $\mathcal{F}_{\alpha}\left(\mathcal{E}_{\xi_{1}}, \mathcal{E}_{\xi_{2}}\right)=F_{\alpha}\left(\xi_{1}, \xi_{2}\right)$, for all $\alpha \in(0,1)$. It is natural to ask if the contrary also holds, that is, if for any channels $\mathcal{E}_{i}$ one can find some processor and programming states $\xi_{i}, i=1$ and 2, respectively, such that $F_{\alpha}\left(\xi_{1}, \xi_{2}\right)=\mathcal{F}_{\alpha}\left(\mathcal{E}_{1}, \mathcal{E}_{2}\right)$ ? It turns out that the answer in this case is negative. To confirm this, we recall that the programming states $\xi_{i} \in \mathcal{S}\left(\mathcal{H}_{E}\right), i=1,2$, resulting into a unitary and any other different extremal channel are necessarily orthogonal to each other, that is, in particular, $F_{1 / 2}\left(\xi_{1}, \xi_{2}\right)=0$; see Ref. [14, Prop. 8]. It is, however, possible to find a unitary channel $\mathcal{U}$ and an extremal channel $\mathcal{E} \neq \mathcal{U}$ with $\mathcal{F}_{1 / 2}(\mathcal{U}, \mathcal{E}) \neq 0$. Indeed, let us choose $\mathcal{U}$ as the one-qubit identity channel and for any $\varrho_{\vec{m}} \in \mathcal{S}\left(\mathbb{C}^{2}\right)$ define $\mathcal{E}\left(\varrho_{\vec{m}}\right)=\frac{1}{2}\left(\mathbb{1}+m_{2} \sigma_{2}\right)$. The extremality of $\mathcal{E}$ can be verified by using the results of Ref. [34], and furthermore using inequalities found in Ref. [35] one can show that

$$
\begin{aligned}
& \mathcal{F}_{1 / 2}(\mathcal{U}, \mathcal{E}) \\
& \geqslant \inf _{\substack{\|\vec{n}\| \leqslant 1,\|\vec{m}\| \leqslant 1}} \frac{\operatorname{tr}\left[\varrho_{\vec{n}} \mathcal{E}\left(\varrho_{\vec{m}}\right)\right]}{\operatorname{tr}\left[\varrho_{\vec{n}} \varrho_{\vec{m}}\right]+\sqrt{\left(1-\operatorname{tr}\left[\varrho_{\vec{n}}^{2}\right]\right)\left(1-\operatorname{tr}\left[\varrho_{\vec{m}}^{2}\right]\right)}} \\
& =1 / 2 .
\end{aligned}
$$

We conclude that inequality (16) fails to be tight in general.

Nevertheless, the channel $\alpha$ fidelity $\mathcal{F}_{\alpha}$ is a genuinely important figure of merit in quantum programming, as we will see in the following. According to the previous proposition, programming $N$ different unitary channels requires $\operatorname{dim}\left(\mathcal{H}_{E}\right) \geqslant N$. The processor's dimension may, however, be lowered if some error is accepted. Let us denote $d=\operatorname{dim}\left(\mathcal{H}_{S}\right)$. Suppose we wish to program the approximate unitary channels $\mathcal{E}_{U_{i}}(\varrho)=(1-\varepsilon) \mathcal{U}_{j}(\varrho)+\varepsilon \frac{1}{d} \mathbb{1}_{\mathcal{H}_{s}}$ with programming vectors $\left|\phi_{i}\right\rangle$, respectively, where $U_{i}, \quad i=1, \ldots, N$ are unequal unitaries and $\varepsilon \in[0,1]$ describes a fixed error rate. From Ref. [35], we find that $F_{1 / 2}\left(\mathcal{E}_{U_{j}}\left(\varrho_{1}\right), \mathcal{E}_{U_{k}}\left(\varrho_{2}\right)\right) \leqslant$ $\operatorname{tr}\left[\mathcal{E}_{U_{j}}\left(\varrho_{1}\right) \mathcal{E}_{U_{k}}\left(\varrho_{2}\right)\right]+\sqrt{\left(1-\operatorname{tr}\left[\mathcal{E}_{U_{j}}\left(\varrho_{1}\right)^{2}\right]\right)\left(1-\operatorname{tr}\left[\mathcal{E}_{U_{k}}\left(\varrho_{2}\right)^{2}\right]\right)}$ Choosing $\varrho_{i}=\left|\varphi_{i}\right\rangle\left\langle\varphi_{i}\right|, i=1$ and 2 simplifies this inequality yielding $\frac{F_{1 / 2}\left(\mathcal{E}_{U_{j}}\left(\varrho_{1}\right), \mathcal{E}_{U_{k}}\left(\varrho_{2}\right)\right)}{F_{1 / 2}\left(\varrho_{1}, \varrho_{2}\right)} \leqslant \frac{(1-\varepsilon)^{2}\left|\left\langle U_{j} \varphi_{1} \mid U_{k} \varphi_{2}\right\rangle\right|^{2}}{\left|\left\langle\varphi_{1} \mid \varphi_{2}\right\rangle\right|}+\frac{(2-\varepsilon) \varepsilon}{\left|\left\langle\varphi_{1} \mid \varphi_{2}\right\rangle\right|}$. In particular, choosing $\varphi_{2}$ as in Eq. (17) for $U=U_{j}^{\dagger} U_{k}$ implies

$$
\begin{aligned}
F_{1 / 2}\left(\phi_{j}, \phi_{k}\right) & \leqslant \inf _{\left|\varphi_{1}\right\rangle} \frac{(2-\varepsilon) \varepsilon}{\sqrt{1-\left|\left\langle U_{j} \varphi_{1} \mid U_{k} \varphi_{1}\right\rangle\right|^{2}}} \\
& =\frac{(2-\varepsilon) \varepsilon}{\sqrt{1-\inf _{\varrho} F_{1 / 2}\left(\mathcal{U}_{j}(\varrho), \mathcal{U}_{k}(\varrho)\right)}} \doteq g_{j k}(\varepsilon),
\end{aligned}
$$

when $j \neq k$. We may use this bound to solve for the largest set of linearly independent programming vectors using the following result: if $\left|\phi_{j}\right\rangle, j=1, \ldots, K$, are unit vectors and $F_{1 / 2}\left(\phi_{j}, \phi_{k}\right) \leqslant \frac{1}{K-1}$ whenever $j \neq k$, then the vectors $\left|\phi_{j}\right\rangle, j=1, \ldots, K$, are linearly independent [12]. Let us denote with $K_{\varepsilon}$ the largest integer such that $K_{\varepsilon}<$

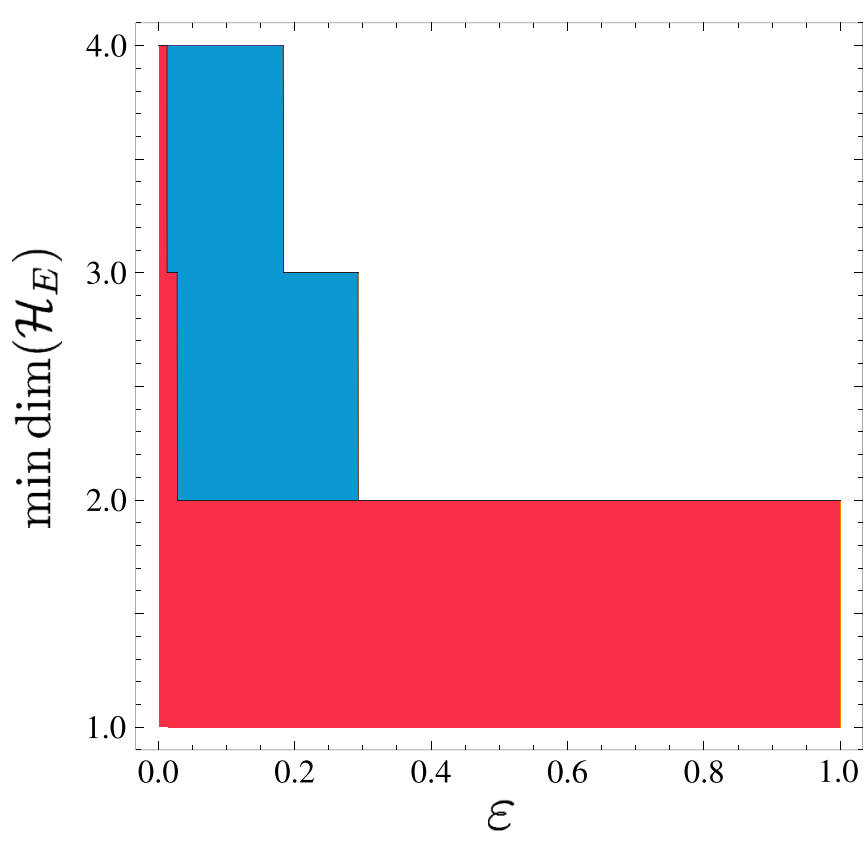

FIG. 2. Dimension of programmable processor in approximate programming. We consider the dimension of a processor capable of implementing the four noisy unitary channels $\mathcal{E}_{i}: \varrho \mapsto(1-$ $\varepsilon) \sigma_{i} \varrho \sigma_{i}^{\dagger}+\varepsilon \frac{1}{2} \mathbb{1}, i=0, \ldots, 3$, in terms of the noise parameter $\varepsilon \in$ $[0,1]$. It may be confirmed that the process fidelity satisfies $F_{\text {proc }}\left(\sigma_{i}\right.$. $\left.\sigma_{i}^{\dagger}, \mathcal{E}_{i}\right) \geqslant 1-\varepsilon$ for all $i=0, \ldots, 3$. Accordingly, an analysis done in Ref. [13] implies that any processor implementing $\mathcal{E}_{i}, i=0, \ldots, 3$, is at least four-dimensional for $0 \leqslant \varepsilon<[3(13+2 \sqrt{42})]^{-1}$, at least three-dimensional for $[3(13+2 \sqrt{42})]^{-1} \leqslant \varepsilon<[2(9+4 \sqrt{5})]^{-1}$ and at least two-dimensional for $[2(9+4 \sqrt{5})]^{-1} \leqslant \varepsilon<1$ excluding the dimensions within the red area. However, our approach results to tighter limits, which we have denoted in blue: the processor is necessarily at least four-dimensional for $0 \leqslant \varepsilon<\frac{1}{3}(3-\sqrt{6})$, at least three-dimensional for $\frac{1}{3}(3-\sqrt{6}) \leqslant \varepsilon<\frac{1}{2}(2-\sqrt{2})$ and at least two-dimensional for $\frac{1}{2}(2-\sqrt{2}) \leqslant \varepsilon<1$.

$\max _{j \neq k \in\{1, \ldots N\}}\left(1 / g_{j k}(\varepsilon)+1\right)$. The previous result then implies that any set of vectors whose size is less than or equal to $K_{\varepsilon}$ is linearly independent. Therefore, if $N \leqslant K_{\varepsilon}$, then all of the programming vectors are linearly independent and $\operatorname{dim}\left(\mathcal{H}_{E}\right) \geqslant N$. On the other hand, if $N>K_{\varepsilon}$, then $\operatorname{dim}\left(\mathcal{H}_{E}\right) \geqslant K_{\varepsilon}[13]$.

As an example, let us consider approximate programming of qubit unitaries $\sigma_{i}, i=0, \ldots, 3$, where $\sigma_{0}=\mathbb{1}_{\mathbb{C}^{2}}$. For all pairs the quantity $\inf _{\varrho} F_{1 / 2}\left(\sigma_{j} \varrho \sigma_{j}, \sigma_{k} \varrho \sigma_{k}\right)$ vanishes. Therefore, according to Eq. (19), the processor in approximate programming of the above unitaries is at least four-dimensional for $0 \leqslant$ $\varepsilon<\frac{1}{3}(3-\sqrt{6})$, at least three-dimensional for $\frac{1}{3}(3-\sqrt{6}) \leqslant$ $\varepsilon<\frac{1}{2}(2-\sqrt{2})$ and at least two-dimensional for $\frac{1}{2}(2-\sqrt{2}) \leqslant$ $\varepsilon<1$. In Fig. 2, these bounds have been compared to those previously solved in Ref. [13]. It is noteworthy that our approach gives tighter limits for the amount of noise necessary to be present in order to approximately implement the four unitary channels $\sigma_{i}, i=0, \ldots, 3$. 


\section{B. Ruling out incompatible environmental properties}

Let us consider a general thermal environment $\mathcal{H}_{E}$ and two initial states of the environment in different temperatures $T_{1}$ and $T_{2}$. The environmental states therefore attain the Gibbs form $\xi\left(\beta_{i}\right) \doteq \exp \left[-\beta_{i} \mathrm{H}_{E}\right] / Z\left(\beta_{i}, \mathrm{H}_{E}\right), i=$ 1 and 2, where $\beta_{i}=\left(k_{B} T_{i}\right)^{-1}$, where $\mathrm{H}_{E}$ is a Hamiltonian of the environment and $Z\left(\beta_{i}, \mathrm{H}_{E}\right) \doteq \operatorname{tr}\left[\exp \left[-\beta_{i} \mathrm{H}_{E}\right]\right]$ are the corresponding partition functions for $i=1$ and 2 .

Our goal is to exclude some properties of environment inscribed in the spectrum of the Hamiltonian $\mathrm{H}_{E}$ that are incompatible with inequality (15). Since for thermal states $\left[\xi\left(\beta_{1}\right), \xi\left(\beta_{2}\right)\right]=0$, we easily solve

$$
S_{\alpha}\left(\xi\left(\beta_{1}\right) \| \xi\left(\beta_{2}\right)\right)=\frac{1}{\alpha-1}\left(\ln \left[Z\left(\alpha \beta_{1}+(1-\alpha) \beta_{2}, \mathrm{H}_{E}\right)\right]-\alpha \ln \left[Z\left(\beta_{1}, \mathrm{H}_{E}\right)\right]-(1-\alpha) \ln \left[Z\left(\beta_{2}, \mathrm{H}_{E}\right)\right]\right) .
$$

In particular, for all $\alpha \in\left[\frac{1}{2}, 1\right)$, inequality (15) implies the following inequality:

$$
\ln \left[Z\left(\alpha \beta_{1}+(1-\alpha) \beta_{2}, \mathrm{H}_{E}\right)\right]-\alpha \ln \left[Z\left(\beta_{1}, \mathrm{H}_{E}\right)\right]-(1-\alpha) \ln \left[Z\left(\beta_{2}, \mathrm{H}_{E}\right)\right] \leqslant \inf _{t \geqslant 0} \ln \left[\mathcal{F}_{\alpha}\left(\mathcal{E}_{1}^{(t)}, \mathcal{E}_{2}^{(t)}\right)\right] .
$$

In fact, due to the commutativity of the thermal states, we can expand this for $\alpha \in(0,1)$ such that

$$
\ln \left[Z\left(\alpha \beta_{1}+(1-\alpha) \beta_{2}, \mathrm{H}_{E}\right)\right]-\alpha \ln \left[Z\left(\beta_{1}, \mathrm{H}_{E}\right)\right]-(1-\alpha) \ln \left[Z\left(\beta_{2}, \mathrm{H}_{E}\right)\right] \leqslant \inf _{t \geqslant 0}\left\{\begin{array}{ll}
\ln \left[\mathcal{F}_{\alpha}\left(\mathcal{E}_{2}^{(t)}, \mathcal{E}_{1}^{(t)}\right)\right], & \text { for } \alpha \in\left(0, \frac{1}{2}\right) \\
\ln \left[\mathcal{F}_{\alpha}\left(\mathcal{E}_{1}^{(t)}, \mathcal{E}_{2}^{(t)}\right)\right], & \text { for } \alpha \in\left(\frac{1}{2}, 1\right)
\end{array} .\right.
$$

Let us consider the implications of the above inequalities. Assume that it is possible to prepare the environment in two different known temperatures and perform the full-process tomography of the two induced dynamics. This enables us to determine the values of $\mathcal{F}_{\alpha}$ in the right-hand side of inequality (22). On the other hand, any hypothesis about the Hamiltonian of the environment determines the left-hand side of the inequality. If the inequality is not satisfied, the hypothesized Hamiltonian of the system can be ruled out as incompatible with the induced pair of dynamics. We highlight that in order to do so neither the system-environment coupling nor the Hamiltonian of the system needs to be specified.

As a demonstration of the power of this method, let us consider a specific example of a qubit system coupled to an environment consisting of a single harmonic oscillator: such a situation could occur, for instance, when a two-level atom is passing through an optical cavity including only a single quantized mode. Assume that the mechanism of interaction is unknown as well as the oscillator frequency which we nevertheless wish to determine. Mathematically, we only know that the Hamiltonian of the environment is of the form $\mathrm{H}_{E}=\omega\left(b^{\dagger} b+\frac{1}{2} \mathbb{1}\right)$. Choosing different values for $\omega$ one plots the dashed coloured curves in Fig. 3 given by the left-hand side of inequality (22). For a concrete example of the (exactly solvable) reduced dynamics giving the right-hand side of inequality (22) and the solid black curve in Fig. 3, we consider the interaction model of the form

$$
\begin{aligned}
\mathrm{H} & =\mathrm{H}_{S}+\mathrm{H}_{E}+\mathrm{H}_{I} \\
& =\frac{\omega_{0}}{2} \sigma_{3}+\omega\left(b^{\dagger} b+\frac{1}{2} \mathbb{1}\right)+\sigma_{3} \otimes\left(g b^{\dagger}+g^{*} b\right)
\end{aligned}
$$

analyzed in Ref. [1]. One can see that the inequality forbids frequency values greater than 3.1 times the real frequency $\omega$. For details, we refer the reader to Appendix B.

\section{Quantum thermometry}

Let us consider the situation of the previous example from a different perspective. Suppose now that a thermal environment described by a single harmonic oscillator with Hamiltonian $\mathrm{H}_{E}=\omega\left(b^{\dagger} b+\frac{1}{2} \mathbb{1}\right)$ is initially prepared at calibration temperature $T_{0}=0$, that is $\xi\left(\beta_{0}=\infty\right)=|0\rangle\langle 0|$. Let us assume we are tasked with probing another temperature $T$ of the environment. The $\alpha$-Rényi divergence between the states $\xi\left(\beta_{0}\right)$ and $\xi\left(\beta=1 /\left(k_{B} T\right)\right)$ now reads $S_{\alpha}\left(\xi\left(\beta_{0}\right) \| \xi(\beta)\right)=$ $\frac{1-\alpha}{\alpha-1}\left(-\frac{\beta \hbar \omega}{2}-\ln \left[Z\left(\beta, \mathrm{H}_{E}\right)\right]\right)$ and therefore after some simple algebra we solve from inequality (5) a lower bound for the

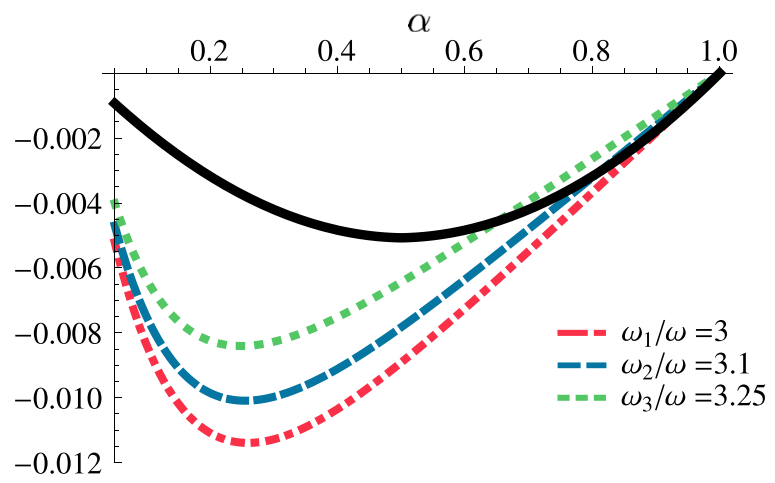

FIG. 3. Excluding the frequency values of a harmonic oscillator. (In this example, we have fixed $\hbar=1=k_{B}$.) We prepare the environment in two temperatures and measure the induced dynamics, which gives us right-hand side of inequality (22) (black line). The three dashed colored lines correspond to the left-hand side of inequality (22) with different choices for $\omega_{k}, k=1,2$, and 3, in units of the actual frequency $\omega$. Knowing the two temperatures of the environment $\left(T_{1} / \omega=0.25\right)$ and $\left(T_{2} / \omega=0.75\right)$, we notice that some frequencies $\left(\omega_{1} / \omega=3\right)$ are compatible with inequality (22) while others $\left(\omega_{2} / \omega=3.1\right.$ and $\left.\omega_{3} / \omega=3.25\right)$ violate it. In fact, we see that inequality (22) is violated for all frequencies larger than $\omega_{2} / \omega=3.1$, and thus $\omega_{2} / \omega=3.1$ is the crossover between the compatible and incompatible frequencies. As a probe we use a qubit and the induced dynamics result from an exactly solvable model described in Ref. [1]. The optimal initial state(s) of the qubit are $\varrho=|+\rangle\langle+|$ with $|+\rangle=\frac{1}{\sqrt{2}}(|0\rangle+|1\rangle)$ being the eigenstate of Pauli operator $\sigma_{1}$; for details, see the Appendix B. 
temperature

$$
\frac{k_{B} T}{\hbar \omega} \geqslant-1 \times \begin{cases}{\left[\ln \left[1-\inf _{t \geqslant 0} \mathcal{F}_{\alpha}\left(\mathcal{E}_{T}^{(t)}, \mathcal{E}_{0}^{(t)}\right)^{\frac{1}{\alpha}}\right]\right]^{-1},} & \alpha \in\left(0, \frac{1}{2}\right) \\ {\left[\ln \left[1-\inf _{t \geqslant 0} \mathcal{F}_{\alpha}\left(\mathcal{E}_{0}^{(t)}, \mathcal{E}_{T}^{(t)}\right)^{\frac{1}{1-\alpha}}\right]\right]^{-1},} & \alpha \in\left(\frac{1}{2}, 1\right)\end{cases}
$$

for all $T \geqslant 0$. It can be confirmed that with temperatures lower than a limiting temperature $\left(k_{B} T\right) /(\hbar \omega) \lesssim 1.03$, the term $-\ln \left[Z\left(\beta, \mathrm{H}_{E}\right)\right]=\frac{\beta \hbar \omega}{2}+\ln \left[1-e^{-\beta \hbar \omega}\right]$ is non-negative, from which, by using inequality (5), we may also obtain an upper bound for the temperature:

$$
\frac{k_{B} T}{\hbar \omega} \leqslant-1 / 2 \times \begin{cases}{\left[\ln \left[\inf _{t \geqslant 0} \mathcal{F}_{\alpha}\left(\mathcal{E}_{T}^{(t)}, \mathcal{E}_{0}^{(t)}\right)^{\frac{1}{\alpha}}\right]\right]^{-1},} & \alpha \in\left(0, \frac{1}{2}\right) \\ {\left[\ln \left[\inf _{t \geqslant 0} \mathcal{F}_{\alpha}\left(\mathcal{E}_{0}^{(t)}, \mathcal{E}_{T}^{(t)}\right)^{\frac{1}{1-\alpha}}\right]\right]^{-1},} & \alpha \in\left(\frac{1}{2}, 1\right)\end{cases}
$$

Since there is no dependency on the parameter $\alpha$ on the left-hand side of the above inequalities, we optimize over this parameter. The quantities on the right-hand side of the inequalities above can be solved (numerically) after measuring the dynamics; these observations allow us to estimate the unknown temperature $T$.

Inequalities (24) and (25) may, unfortunately, be difficult to evaluate in general due to double optimization with respect to states and therefore a more accessible form is desirable. To achieve this, we use the fact that $\mathcal{F}_{\alpha}\left(\mathcal{E}_{1}, \mathcal{E}_{2}\right) \leqslant$ $\inf _{\varrho} F_{\alpha}\left(\mathcal{E}_{1}(\varrho), \mathcal{E}_{2}(\varrho)\right)$, for all $\alpha \in(0,1)$. Since $S_{\alpha}(\cdot \| \cdot)$ is monotonically increasing in $\alpha$ [20], that is, $S_{\alpha}\left(\varrho_{1} \| \varrho_{2}\right) \leqslant$ $S_{\alpha^{\prime}}\left(\varrho_{1} \| \varrho_{2}\right)$ whenever $\alpha \leqslant \alpha^{\prime}$, and since the logarithm function is monotonically increasing, we conclude that $F_{\alpha}\left(\varrho_{1}, \varrho_{2}\right)^{\frac{1}{1-\alpha}}$ monotonically decreases in $\alpha$. On the other hand, since for all positive semidefinite linear operators $A, B$ and numbers $q \geqslant 0, r \geqslant 1$ the Araki-Lieb-Thirring inequality [36,37] states that $\operatorname{tr}\left[\left(B^{1 / 2} A B^{1 / 2}\right)^{r q}\right] \leqslant \operatorname{tr}\left[\left(B^{r / 2} A^{r} B^{r / 2}\right)^{q}\right]$ holds and since $F_{\alpha}\left(\varrho_{1}, \varrho_{2}\right)$ may be recast in the form ${ }^{2} F_{\alpha}\left(\varrho_{1}, \varrho_{2}\right)=$ $\operatorname{tr}\left[\left(\varrho_{1}^{1 / 2} \varrho_{2}^{(1-\alpha) / \alpha} \varrho_{1}^{1 / 2}\right)^{\alpha}\right]$, we have

$$
\begin{aligned}
F_{\alpha}\left(\varrho_{1}, \varrho_{2}\right) & =\operatorname{tr}\left[\left(\varrho_{1}^{1 / 2} \varrho_{2}^{(1-\alpha) / \alpha} \varrho_{1}^{1 / 2}\right)^{\frac{\alpha}{1-\alpha}(1-\alpha)}\right] \\
& \leqslant \operatorname{tr}\left[\left(\varrho_{1}^{\alpha / 2(1-\alpha)} \varrho_{2} \varrho_{1}^{\alpha / 2(1-\alpha)}\right)^{1-\alpha}\right] \\
& =F_{1-\alpha}\left(\varrho_{2}, \varrho_{1}\right)
\end{aligned}
$$

and therefore $F_{\alpha}\left(\varrho_{1}, \varrho_{2}\right)^{1 /(1-\alpha)} \leqslant F_{1-\alpha}\left(\varrho_{2}, \varrho_{1}\right)^{1 /(1-\alpha)}$ whenever $\alpha \geqslant 1 / 2\left(\Leftrightarrow r=\frac{\alpha}{1-\alpha} \geqslant 1\right)$. To summarize,

$$
\begin{aligned}
\frac{k_{B} T}{\hbar \omega} & \geqslant-\ln \left[1-\inf _{\varrho, t \geqslant 0} \lim _{\alpha \nearrow 1} F_{\alpha}\left(\mathcal{E}_{0}^{(t)}(\varrho), \mathcal{E}_{T}^{(t)}(\varrho)\right)^{\frac{1}{1-\alpha}}\right]^{-1} \\
& =-\ln \left[1-e^{-\sup _{\varrho, t \geqslant 0} S_{1}\left(\mathcal{E}_{0}^{(t)}(\varrho) \| \mathcal{E}_{T}^{(t)}(\varrho)\right)}\right]^{-1}
\end{aligned}
$$

for $\quad T \geqslant 0$, where $\quad \lim _{\alpha{ }_{1}} S_{\alpha}\left(\varrho_{1} \| \varrho_{2}\right)=S_{1}\left(\varrho_{1} \| \varrho_{2}\right) \doteq$ $\operatorname{tr}\left[\varrho_{1}\left(\ln \varrho_{1}-\ln \varrho_{2}\right)\right]$ is the Kullback-Leibler divergence [20]. Similarly, for the upper bound of temperature one derives

$$
\begin{aligned}
\frac{k_{B} T}{\hbar \omega} & \leqslant-1 / 2 \ln \left[\inf _{\varrho, t \geqslant 0} \lim _{\alpha \nearrow 1} F_{\alpha}\left(\mathcal{E}_{0}^{(t)}(\varrho), \mathcal{E}_{T}^{(t)}(\varrho)\right)^{\frac{1}{1-\alpha}}\right]^{-1} \\
& =\left(2 \sup _{\varrho, t \geqslant 0} S_{1}\left(\mathcal{E}_{0}^{(t)}(\varrho) \| \mathcal{E}_{T}^{(t)}(\varrho)\right)\right)^{-1}
\end{aligned}
$$

when $0 \leqslant\left(k_{B} T\right) /(\hbar \omega) \lesssim 1.03$.

\footnotetext{
${ }^{2}$ This follows from the fact that the operators $X X^{\dagger}$ and $X^{\dagger} X$ have the same nonzero eigenvalues, and therefore $\operatorname{tr}\left[\left(X X^{\dagger}\right)^{\alpha}\right]=\operatorname{tr}\left[\left(X^{\dagger} X\right)^{\alpha}\right]$.
}

In Fig. 4, we have plotted $\left(k_{B} T\right) /(\hbar \omega)$ and the optimal bounds given by the right-hand sides of inequalities (27) and (28) for the resonant Jaynes-Cummings model, which describes a two-level atom interacting with a single quantized mode of radiation in an optical cavity [1,38]; for details we refer the reader to Appendix $C$. In the figure, we have chosen the initial state of the qubit probe as $\varrho=|+\rangle\langle+|$, since numerics suggest that it leads to the optimal bounds.

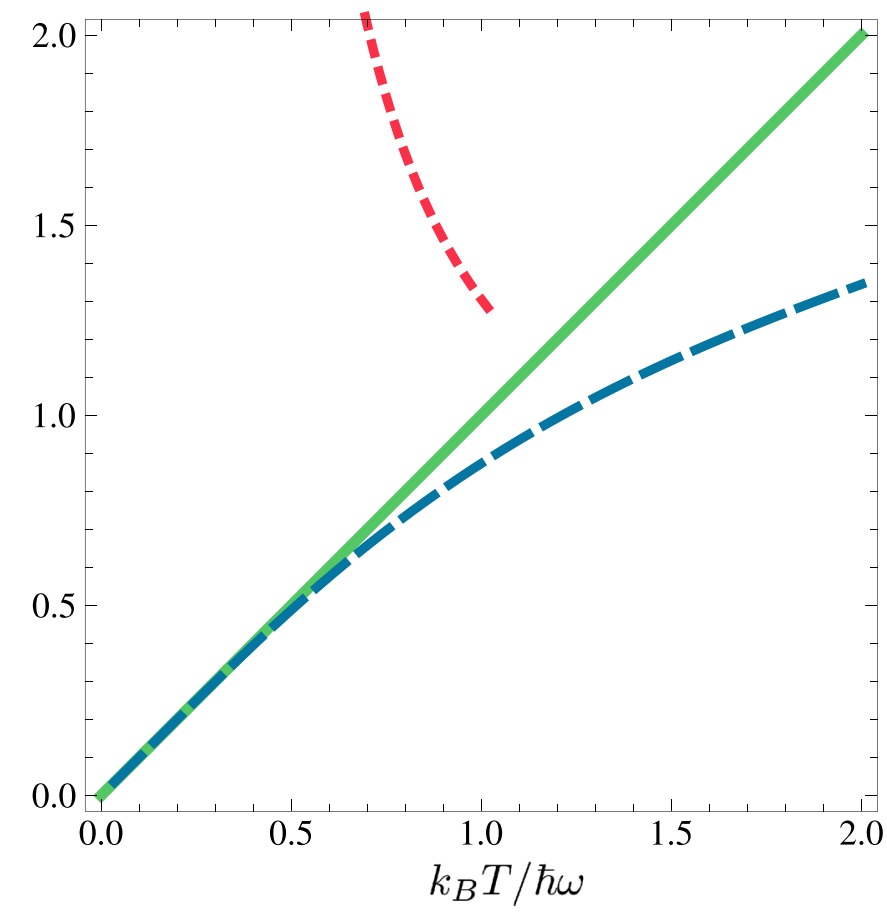

FIG. 4. Estimation of temperature. We assume that the environment is prepared in two different temperatures $\left[\left(k_{B} T_{0}\right) /(\hbar \omega)=0\right.$ and $\left.\left(k_{B} T\right) /(\hbar \omega)\right]$ and measure the induced dynamics. The green line represents the actual temperature $\left(k_{B} T\right) /(\hbar \omega)$. From inequality (27), we solve a lower bound of $\left(k_{B} T\right) /(\hbar \omega)$, plotted above as the blue dashed curve. The red dotted curve corresponds to the upper bound of the temperature valid whenever $\left(k_{B} T\right) /(\hbar \omega) \lesssim 1.03$ given by inequality (28). The system that we use for probing the temperature is a qubit and the dynamics are due to Jaynes-Cummings model in the resonant case (see Ref. [38]). The initial state of the qubit that we have used for in the plot is $\varrho=|+\rangle\langle+|$, since numerics suggest that it leads to the optimal bounds. 
A standard procedure in (quantum) thermometry is to bring the thermometer into contact with a bath, let it thermalize, and then read out the temperature. In the quantum scenario, we say that a qubit probe has thermalized with the bath if any initial state $\varrho$ of the probe evolves in the long time limit $t \rightarrow \infty$ into the equilibrium Gibbs state $\varrho \rightarrow \varrho_{\text {eq }} \doteq(1-p(T))|0\rangle\langle 0|+$ $p(T)|1\rangle\langle 1|$, where $p(T)=\left[1+\exp \left(\hbar \omega / k_{B} T\right)\right]^{-1}$. Quantum thermometry protocols [39-42] and optimality of initial probe states [43] have been studied for both fully and partially thermalizing probes. To our best knowledge, all preexisting protocols are based on knowing the exact form of the coupling. Also the optimal initial probe states have been solved in the partially thermalized probes only for fixed system-probe couplings.

In the case of fully thermalized probes, calculating the $\alpha$ fidelity becomes particularly easy: $\lim _{t \rightarrow \infty} \mathcal{F}_{\alpha}\left(\mathcal{E}_{0}^{(t)}, \mathcal{E}_{T}^{(t)}\right)=$ $(1-p(T))^{1-\alpha}$. Accordingly, our protocol sets the limits

$$
-[\ln [p(T)]]^{-1} \leqslant \frac{k_{B} T}{\hbar \omega} \leqslant-1 / 2[\ln [1-p(T)]]^{-1},
$$

with the upper bound valid whenever $0 \leqslant\left(k_{B} T\right) /(\hbar \omega) \lesssim 1.03$. It is noteworthy that due to the special form of the state $\varrho_{\text {eq }}$ the temperature $T$ could be solved analytically from the state occupations, which shows that our protocol is not the optimal one for thermometry under the assumption of thermalization. However, there is no a priori need to require thermalization of the probe with the bath in our protocol. This is an important advantage, since thermalization itself in a fully quantum scenario may not be a well-defined concept due to the ever present quantum fluctuations and the long time scales needed compared to the survival time of the quantum properties of the bath.

We wish to emphasize that knowing the specific system environment coupling is not required in the above two parameter estimation protocols $\mathrm{B}$ and $\mathrm{C}$ : to the best of our knowledge, such probing approaches independent of the description of the coupling have never been proposed before. While the precisions of the frequency estimation and thermometer are clearly not optimal, these examples serve as proofs-of-principle demonstrations of the ability to extract information on certain physical quantities by means of our approach, making only minimal assumptions on the microscopic details of the system to be probed. From a broader point of view, the two protocols $\mathrm{B}$ and $\mathrm{C}$ could be applied to estimate, not only the frequency and temperature, but also other physical quantities of a system of interest. The task to probe some of such quantities could be unattainable "conventionally," since the description of the coupling model between the system and the probe is not either accurately known or is borderline totally missing. An ultimate example of such case is the quantum gravity, since no commonly agreed model of gravitational interaction in quantum regime exists.

\section{Bounds for the Loschmidt echo}

In our last application, we change the perspective altogether and consider a different implication of inequality (8). We show how from the $\alpha$ fidelity of the initial environmental states we can infer certain properties of the induced dynamics, such as information back-flow (non-Markovianity). Importantly, back-flow of information can be used to protect and restore vital quantum properties of the system subjected to detrimental noise [44-46].

As an example, we concentrate on one-qubit dephasing channels arising from system environment interaction. Although the method of this section holds generally for such channels, we demonstrate the power of the protocol by focusing on a specific situation with known solutions only for certain initial states of environment: a qubit transversely coupled to its environment, which is an Ising spin chain in a transverse field. The total evolution of such a composite system is governed by the Hamiltonian $\mathrm{H}(\lambda, \delta)=-J \Sigma_{j}\left(\sigma_{3}^{(j)} \otimes \sigma_{3}^{(j+1)}+\right.$ $\left.\lambda \sigma_{1}^{(j)}+\delta|e\rangle\langle e| \otimes \sigma_{1}^{(j)}\right)$. Here the parameters $J, \lambda$, and $\delta$ characterize the strength of the nearest-neighbor interactions in the Ising chain, its coupling to a transverse field and the coupling between system and environment, respectively $[47,48]$. It is known that the initial environmental state $|\phi\rangle$ induces dynamics corresponding to a pure dephasing channel $\mathcal{E}_{\phi}^{(t)}(\varrho)=p_{\phi}(t) \varrho+\left[1-p_{\phi}(t)\right] \sigma_{3} \varrho \sigma_{3}$ with the probability $p_{\phi}(t)$ given by

$$
p_{\phi}(t)=\frac{1}{2}\left(1+\sqrt{L_{\phi}(\lambda, t)}\right)
$$

where $L_{\phi}(\lambda, t)=\left|\left\langle\phi \mid e^{i \mathrm{H}(\lambda, 0) t} e^{-i \mathrm{H}(\lambda+\delta, 0) t} \phi\right\rangle\right|^{2}$ is the Loschmidt echo corresponding to the state $|\phi\rangle$. Despite the apparent simplicity, finding an analytical expression of $L_{\phi}(\lambda, t)$ is a difficult task for a general initial state $|\phi\rangle$. In fact, to our best knowledge, the only known analytical solution exists for the ground state $\left|\phi_{0}\right\rangle$ of the Hamiltonian $\mathrm{H}(\lambda, 0)$ for which one obtains

$$
L_{\phi_{0}}(\lambda, t)=\Pi_{k>0}\left[1-\sin ^{2}\left(2 \alpha_{k}\right) \sin ^{2}\left(\varepsilon_{k} t\right)\right],
$$

where $\alpha_{k}$ are the Bogoliubov angles and $\varepsilon_{k}$ the single quasiparticle excitation energies of the system with the qubit in the excited state $|e\rangle[47,48]$.

Using inequality (8) allows us to give an estimate for the Loschmidt echo $L_{\phi}(\lambda, t)$ that is valid for any state $|\phi\rangle$. Indeed, fixing an initial state $\varrho=|+\rangle\langle+|$ of the qubit, $|+\rangle=\frac{1}{\sqrt{2}}(|0\rangle+$ |1)) being the eigenstate of $\sigma_{1}$, we can solve

$$
\mathcal{E}_{\phi}^{(t)}(\varrho)=\frac{1}{2}\left(\begin{array}{cc}
1 & \sqrt{L_{\phi}(\lambda, t)} \\
\sqrt{L_{\phi}(\lambda, t)} & 1
\end{array}\right) .
$$

It can be concluded that

$$
\begin{aligned}
F_{1 / 2}\left(\phi_{0}, \phi\right) \leqslant & F_{1 / 2}\left(\mathcal{E}_{\phi_{0}}^{(t)}(\varrho), \mathcal{E}_{\phi}^{(t)}(\varrho)\right) \\
= & \frac{1}{2} \sqrt{\left(1-\sqrt{L_{\phi_{0}}(\lambda, t)}\right)\left(1-\sqrt{L_{\phi}(\lambda, t)}\right)} \\
& +\frac{1}{2} \sqrt{\left(1+\sqrt{L_{\phi_{0}}(\lambda, t)}\right)\left(1+\sqrt{L_{\phi}(\lambda, t)}\right)},
\end{aligned}
$$

from which an estimate for $L_{\phi}(\lambda, t)$ in terms of $F_{1 / 2}\left(\phi_{0}, \phi\right)$ may be solved. In Fig. 5, we have presented the results for three different values of the strength of the transverse field $\lambda$ fixing the parameters $\delta=0.1, J=1$ and the number of spins $N=4000$. In particular, we see that for those $\lambda$ resulting to revivals in Loschmidt echo in the ground state $\left|\phi_{0}\right\rangle$, after a certain limiting value of $F_{\text {lim }} \leqslant F_{1 / 2}\left(\phi_{0}, \phi\right)$ such revivals are guaranteed for the state $|\phi\rangle$ also. The revivals of Loschmidt echo are linked to revivals of coherences via Eq. (32) and 

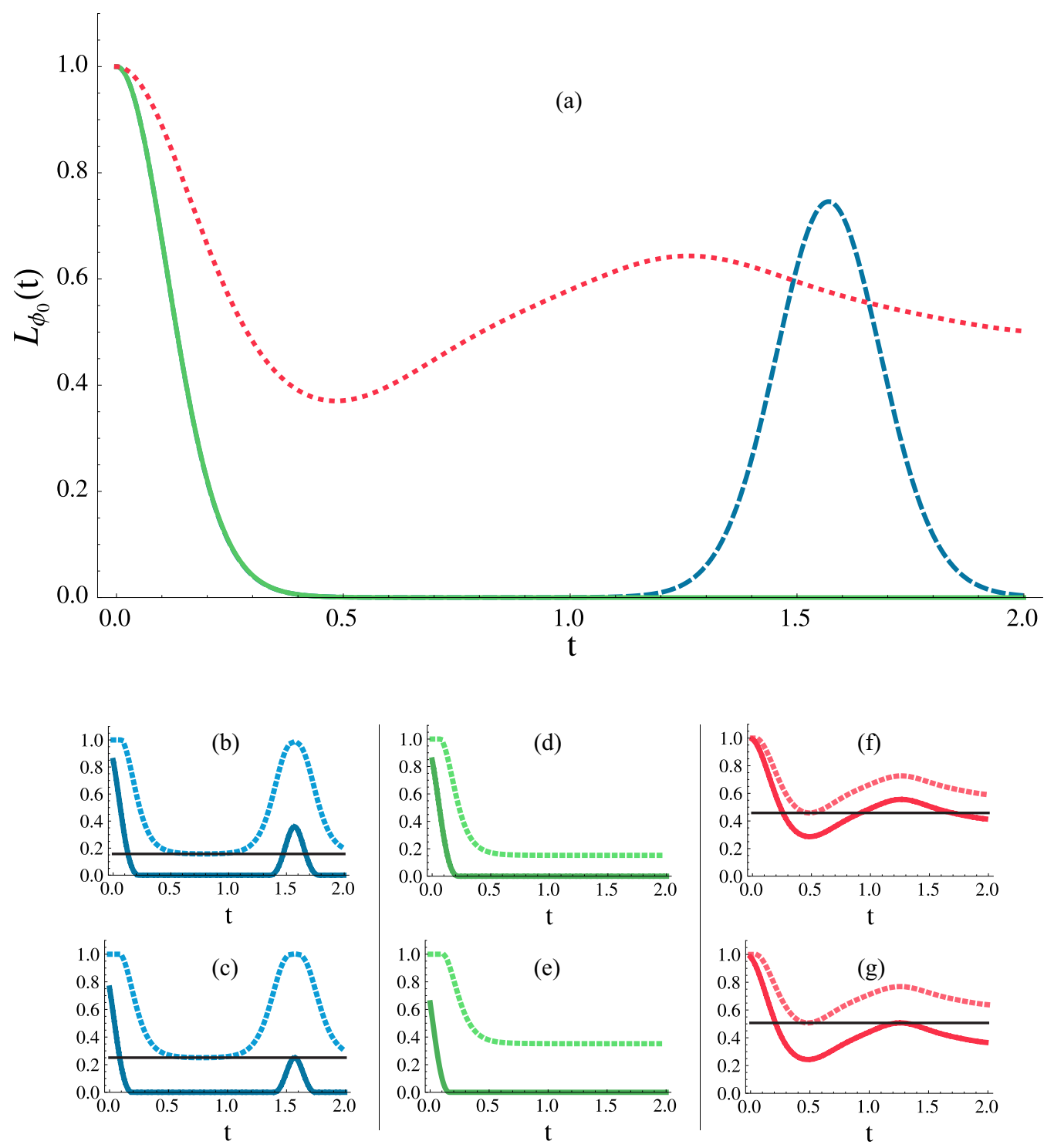

FIG. 5. Bounding the Loschmidt echo. (a) The dynamics of the Loschmidt echo induced by the environmental ground state $\phi_{0}$. The parameter values of the Hamiltonian $\mathrm{H}(\lambda)$ are set as $J=1, \delta=0.1$, and $N=4000$. The blue dashed line corresponds to the choice $\lambda=0.01$, the green solid line corresponds to the critical value $\lambda=0.9$ and the red dotted line corresponds to the value $\lambda=1.8$. (b) $-(\mathrm{g})$ The dynamics of the upper and lower bound of the unknown Loschmidt echo are represented by the curves in lighter and darker hue of the corresponding color choices of the parameter $\lambda$, respectively. The black solid line indicates the level of the first local minimum of the upper bound. In (b) and (f), the lower bounds cross the black line after the local minimum of the upper bound, which implies revivals in the Loschmidt echo and thus information back-flow. (b) $\lambda=0.01$ and $F_{1 / 2}\left(\phi_{0}, \phi\right)=0.98$. (c) $\lambda=0.01$ and $F_{1 / 2}\left(\phi_{0}, \phi\right)=0.966675$. (d) $\lambda=0.9$ and $F_{1 / 2}\left(\phi_{0}, \phi\right)=0.98$. (e) $\lambda=0.9$ and $F_{1 / 2}\left(\phi_{0}, \phi\right)=0.95$. (f) $\lambda=1.8$ and $F_{1 / 2}\left(\phi_{0}, \phi\right)=0.999$. (g) $\lambda=1.8$ and $F_{1 / 2}\left(\phi_{0}, \phi\right)=0.99761$.

hence indicate back-flow of information from environment to system.

It should be pointed out, that the upper and lower bounds of the Loschmidt echo $L_{\phi}$ could be made tighter by considering general $\alpha$ fidelities in the above inequality (33). However, since solving $L_{\phi}$ from inequality (33) in such a general situation can only be done numerically, we have left this examination as a topic for future investigation.

\section{CONCLUSIONS AND OUTLOOK}

In this paper, we have introduced a family of $\alpha$ fidelities of quantum channels. We also have derived an inequality between the $\alpha$ fidelity of the initial states of the environment and the $\alpha$ fidelity of the corresponding induced dynamics: $F_{\alpha}\left(\xi_{1}, \xi_{2}\right) \leqslant \mathcal{F}_{\alpha}\left(\mathcal{E}_{1}^{(t)}, \mathcal{E}_{2}^{(t)}\right), \alpha \in[1 / 2,1)$. From a more practical viewpoint, the inequality was then considered in the context of four different applications: (A) quantum programming, (B) discrimination of environmental properties, (C) quantum thermometry, and in (D) deriving bounds for the Loschmidt echo of a general (pure) initial state of the spin-chain.

In a nutshell, the general procedure of estimating an unknown $\xi_{2}$ requires (1) the possibility to prepare the environment in a known "calibration" state $\xi_{1}$ and (2) the possibility to compare the probe dynamic $\mathcal{E}_{1}^{(t)}$ and $\mathcal{E}_{2}^{(t)}$ induced by these states. To apply our framework to quantum probing 
experimentally, as proposed in examples B-D, the experimenter needs first to perform quantum process tomography and then numerical optimization for all times, which may be extremely difficult. However, the starting point underlying behind our results is inequality (8), which shows that nontrivial bounds for $F_{\alpha}\left(\xi_{1}, \xi_{2}\right)$ may be found for plethora of choices of initial probe states $\varrho_{1}$ and $\varrho_{2}$; the notion of $\alpha$ fidelity of dynamics was developed as the best achievable resolution of this inequality. In particular, choosing $\varrho_{i}=\varrho, i=1$ and 2 , simplifies inequality (8) to

$$
F_{\alpha}\left(\xi_{1}, \xi_{2}\right) \leqslant F_{\alpha}\left(\mathcal{E}_{1}^{(t)}(\varrho), \mathcal{E}_{2}^{(t)}(\varrho)\right) \leqslant 1,
$$

for all $\varrho \in \mathcal{S}\left(\mathcal{H}_{S}\right)$ and $\alpha \in[1 / 2,1)$ and any given time $t \in$ $[0, \infty)$. Although the resulting upper bound $F_{\alpha}\left(\mathcal{E}_{1}(\varrho), \mathcal{E}_{2}(\varrho)\right)$ may not be the optimal one, any choice of the initial state of $\varrho \in \mathcal{S}\left(\mathcal{H}_{S}\right)$ allows one to extract some information of system of interest via the protocols described above. In addition, the full process tomography is now reduced from the full channel tomography to mere state tomography of the evolved states of the probe $\mathcal{E}_{1}(\varrho)$ and $\mathcal{E}_{2}(\varrho)$.

In our theoretical framework, the introduced fidelities are based on the definition of the $\alpha$-Rényi divergence, given in Eq. (4), mainly because of its connection to the Uhlmann fidelity for $\alpha=1 / 2$. However, as we mentioned before, there exists another quantum divergence often encountered in the literature, namely $\widetilde{S}_{\alpha}$ defined in Eq.(3). It is known that also $\widetilde{S}_{\alpha}$ satisfies both the properties (S1)-(S3) and the data processing inequality (S4) for all $\alpha \in(0,1)$ [20]. Hence, also for $\widetilde{F}_{\alpha}\left(\varrho_{1}, \varrho_{2}\right) \doteq \operatorname{tr}\left[\varrho_{1}^{\alpha} \varrho_{2}^{1-\alpha}\right]$, we have $\widetilde{F}_{\alpha}\left(\varrho_{1}, \varrho_{2}\right) \widetilde{F}_{\alpha}\left(\xi_{1}, \xi_{2}\right) \leqslant$ $\widetilde{F}_{\alpha}\left(\mathcal{E}_{1}^{(t)}\left(\varrho_{1}\right), \mathcal{E}_{2}^{(t)}\left(\varrho_{2}\right)\right), \alpha \in(0,1)$. Furthermore, for commuting states $\left[\varrho_{1}, \varrho_{2}\right]=0$ the quantities $S_{\alpha}$ and $\widetilde{S}_{\alpha}$ coincide [20]. Although it is known that $\widetilde{F}_{\alpha} \leqslant F_{\alpha}$, since $S_{\alpha} \leqslant \widetilde{S}_{\alpha}[21,23]$, an interesting question to investigate in the future is which of the two definitions $\widetilde{F}_{\alpha}$ or $F_{\alpha}$ leads to a tighter inequality between the inducing states and induced dynamics-or whether such optimality even holds in general. It is noteworthy, that the results shown in Figs. $3-5$ are independent of the choice of $\widetilde{F}_{\alpha}$ or $F_{\alpha}$ due to the facts that the time-evolved final states of the probe are commuting in all the three cases as well as the environment states in applications $\mathrm{B}, \mathrm{C}$, and since $\widetilde{F}_{\alpha}$ and $F_{\alpha}$ coincide when comparing pure states in $\mathrm{D}$.

Due to the generality of our formalism, our results can be used in different fields of physics: from quantum information theory to solid state physics, from particle physics to cosmology, from quantum gravity to atomic and molecular physics, and from quantum optics to quantum thermodynamics. Therefore our approach has the potential to pave the way to new fundamental theoretical and experimental discoveries in numerous physical scenarios.

\section{ACKNOWLEDGMENTS}

The authors are grateful to Mário Ziman for his comments on the manuscript. S.M was supported by the EU Collaborative project QuProCS (Grant Agreement 641277) and the Academy of Finland (Project No. 287750). M.T. and H.L. acknowledge financial support from the University of Turku Graduate School (UTUGS) and M.T. additionally acknowledges the hospitality of Mathematical Physics Group in Toruń. G.S. was partially supported by the National Science Centre (Project 2015/17/B/ST2/02026), the Magnus Ehrnrooth Foundation and wishes to acknowledge the hospitality of Turku Quantum Technology Group.

\section{APPENDIX A: EVALUATING $\alpha$ FIDELITY OF QUBIT CHANNELS}

In this section, we present the method that has been used to evaluate the $\alpha$ fidelity of one-qubit channels. First, we simplify the analytic expression to make the numeric optimization more feasible. The density matrices of two arbitrary one-qubit states $\varrho_{k}, k=1$ and 2 , can be written in the Bloch form as

$$
\begin{aligned}
\varrho_{k} & =\frac{1}{2}\left(\mathbb{1}+x_{k} \sigma_{1}+y_{k} \sigma_{2}+z_{k} \sigma_{3}\right) \\
& =\frac{1}{2}\left(\begin{array}{cc}
1+z_{k} & x_{k}-i y_{k} \\
x_{k}+i y_{k} & 1-z_{k}
\end{array}\right),
\end{aligned}
$$

where $x_{k}, y_{k}$, and $z_{k}$ are real numbers satisfying $x_{k}^{2}+y_{k}^{2}+$ $z_{k}^{2} \leqslant 1$ and $\sigma_{1}, \sigma_{2}$, and $\sigma_{3}$ are the Pauli operators. The density matrices can be diagonalized as

$$
\begin{aligned}
\varrho_{k}= & \frac{1}{2 r_{k}\left(r_{k}+z_{k}\right)}\left(\begin{array}{cc}
r_{k}+z_{k} & -\left(x_{k}-i y_{k}\right) \\
x_{k}+i y_{k} & r_{k}+z_{k}
\end{array}\right) \\
& \times\left(\begin{array}{cc}
\frac{1+r_{k}}{2} & 0 \\
0 & \frac{1-r_{k}}{2}
\end{array}\right)\left(\begin{array}{cc}
r_{k}+z_{k} & -\left(x_{k}-i y_{k}\right) \\
x_{k}+i y_{k} & r_{k}+z_{k}
\end{array}\right)^{\dagger},
\end{aligned}
$$

where $r_{k}=\sqrt{x_{k}^{2}+y_{k}^{2}+z_{k}^{2}}$. Now by using Eq. (A2), we can simplify the form of $\varrho_{k}^{\frac{1-\alpha}{\alpha}}$ as

$$
\varrho_{k}^{\frac{1-\alpha}{\alpha}}=\frac{1}{2 r_{k}}\left(\begin{array}{cc}
r_{k} A_{k}^{+}+z_{k} A_{k}^{-} & \left(x_{k}-i y_{k}\right) A_{k}^{-} \\
\left(x_{k}+i y_{k}\right) A^{-} & r_{k} A_{k}^{+}-z_{k} A_{k}^{-}
\end{array}\right),
$$

where $A_{k}^{ \pm}=\left(\frac{1+r_{k}}{2}\right)^{\frac{1-\alpha}{\alpha}} \pm\left(\frac{1-r_{k}}{2}\right)^{\frac{1-\alpha}{\alpha}}$. By using Eq. (A3) and the properties of trace and determinant, we can calculate the trace and determinant of the matrix product $\varrho_{2}^{\frac{1-\alpha}{2 \alpha}} \varrho_{1} \varrho_{2}^{\frac{1-\alpha}{2 \alpha}}$ as

$$
\begin{array}{r}
T:=\operatorname{tr}\left[\varrho_{2}^{\frac{1-\alpha}{2 \alpha}} \varrho_{1} \varrho_{2}^{\frac{1-\alpha}{2 \alpha}}\right]=\frac{1}{2} A_{2}^{+}+\frac{1}{2} A_{2}^{-} \frac{r_{1} r_{2}}{r_{2}}, \\
D:=\operatorname{det}\left(\varrho_{2}^{\frac{1-\alpha}{2 \alpha}} \varrho_{1} \varrho_{2}^{\frac{1-\alpha}{2 \alpha}}\right)=\left(\frac{1-r_{2}^{2}}{4}\right)^{\frac{1-\alpha}{\alpha}}\left(\frac{1-r_{1}^{2}}{4}\right) .
\end{array}
$$

From Eqs. (A4) and (A5), we can write the eigenvalues $\lambda_{+}$ and $\lambda_{-}$of $\varrho_{2}^{\frac{1-\alpha}{2 \alpha}} \varrho_{1} \varrho_{2}^{\frac{1-\alpha}{2 \alpha}}$ as

$$
\lambda_{ \pm}=\frac{1}{2}\left(T \pm \sqrt{T^{2}-4 D}\right) .
$$

Using these eigenvalues, we can diagonalize $\varrho_{2}^{\frac{1-\alpha}{2 \alpha}} \varrho_{1} \varrho_{2}^{\frac{1-\alpha}{2 \alpha}}$ as

$$
\varrho_{2}^{\frac{1-\alpha}{2 \alpha}} \varrho_{1} \varrho_{2}^{\frac{1-\alpha}{2 \alpha}}=U R U^{\dagger}
$$

where $R=\operatorname{diag}\left(\lambda_{+}, \lambda_{-}\right)$and $U$ is the diagonalizing unitary matrix found in the same way as in Eq. (A2). Finally, this allows us to express the $\alpha$ fidelity of two arbitrary one-qubit states, $\varrho_{1}$ and $\varrho_{2}$, as

$$
F_{\alpha}\left(\varrho_{1}, \varrho_{2}\right)=\operatorname{tr}\left[\left(U R U^{\dagger}\right)^{\alpha}\right]=\lambda_{+}^{\alpha}+\lambda_{-}^{\alpha} .
$$


Now we see that, in order to calculate the channel $\alpha$ fidelity of one-qubit channels $\mathcal{E}_{1}$ and $\mathcal{E}_{2}$, it is enough to minimize the quotient

$$
\frac{F_{\alpha}\left(\mathcal{E}_{1}\left(\varrho_{1}\right), \mathcal{E}_{2}\left(\varrho_{2}\right)\right)}{F_{\alpha}\left(\varrho_{1}, \varrho_{2}\right)}=\frac{\tilde{\lambda}_{+}^{\alpha}+\tilde{\lambda}_{-}^{\alpha}}{\lambda_{+}^{\alpha}+\lambda_{-}^{\alpha}}
$$

Here, $\tilde{\lambda}_{ \pm}$are eigenvalues of $\mathcal{E}_{2}\left(\varrho_{2}\right)^{\frac{1-\alpha}{2 \alpha}} \mathcal{E}_{1}\left(\varrho_{1}\right) \mathcal{E}_{2}\left(\varrho_{2}\right)^{\frac{1-\alpha}{2 \alpha}}$ calculated in a similar way from the Bloch coordinates of $\mathcal{E}_{1}\left(\varrho_{1}\right)$ and $\mathcal{E}_{2}\left(\varrho_{2}\right)$, and $\lambda_{ \pm}$are the above-solved eigenvalues of $\varrho_{2}^{\frac{1-\alpha}{2 \alpha}} \varrho_{1} \varrho_{2}^{\frac{1-\alpha}{2 \alpha}}$, where $\varrho_{1}$ and $\varrho_{2}$ are the initial states of the qubits. The quantity to be minimized in Eq. (A9) is a function of six variables $x_{1}, y_{1}, z_{1}, x_{2}, y_{2}$, and $z_{2}$ under the constraints $x_{1}^{2}+y_{1}^{2}+z_{1}^{2} \leqslant 1$ and $x_{2}^{2}+y_{2}^{2}+z_{2}^{2} \leqslant 1$.

\section{Example: the case of pure dephasing channels}

In this example the reduced dynamics is a family of dephasing channels with the same invariance basis for all values of time $t$ and the inverse temperature $\beta=1 / k_{B} T$. Let $\Gamma_{1}(t)$ and $\Gamma_{2}(t)$ be the decoherence rates at time $t$ (the same for both channels) corresponding to $\beta_{1}$ and $\beta_{2}$, respectively. The two dephasing channels $\mathcal{E}_{i}$ are defined via

$$
\begin{aligned}
\varrho & =\frac{1}{2}\left(\begin{array}{cc}
1+z & x-i y \\
x+i y & 1-z
\end{array}\right) \\
\mapsto \mathcal{E}_{i}^{(t)}(\varrho) & =\frac{1}{2}\left(\begin{array}{cc}
1+z & \Gamma_{i}(t)(x-i y) \\
\Gamma_{i}(t)^{*}(x+i y) & 1-z
\end{array}\right) .
\end{aligned}
$$

We have performed the numerical minimization of Eq. (A9) by tools of constrained minimisation. More precisely, we have used the method SLSQP (sequential least square programming) implemented in SCIPY.OPTIMIZE library.

The numerical calculation shows, that the minimum is attained when $\varrho_{1}=\varrho_{2}$ is any pure state maximally unbiased with respect to the invariant basis, for example, $|+\rangle\langle+|=$ $\frac{1}{2}\left(\mathbb{1}+\sigma_{1}\right)$. This observation lets us write the explicit formula for $\alpha$ fidelity of the dynamics:

$$
\begin{aligned}
\mathcal{F}_{\alpha}\left(\mathcal{E}_{1}^{(t)}, \mathcal{E}_{2}^{(t)}\right)= & \left(\frac{1+\Gamma_{2}(t)}{2}\right)^{1-\alpha}\left(\frac{1+\Gamma_{1}(t)}{2}\right)^{\alpha} \\
& +\left(\frac{1-\Gamma_{2}(t)}{2}\right)^{1-\alpha}\left(\frac{1-\Gamma_{1}(t)}{2}\right)^{\alpha} .
\end{aligned}
$$

\section{APPENDIX B}

In application B (ruling out incompatible environmental properties), we consider a system coupled to a thermal environment with Hamiltonian $\mathrm{H}_{E}$ in two different inverse temperatures $\beta_{i}=1 / T_{i}, i=1$ and 2; we have used natural units $\hbar=1=k_{B}$ throughout this section. We showed that the partition functions $Z\left(\beta_{i}, \mathrm{H}_{E}\right)$ of the two environmental states and the corresponding induced dynamics $\mathcal{E}_{i}^{(t)}$ satisfy inequality (22). It was reasoned that some of the properties of the environment inscribed in $\mathrm{H}_{E}$ may be ruled out by measuring the dynamics induced by two different temperatures and by testing which partition functions are (in)compatible with inequality (22). In a particular case of environment consisting of harmonic oscillators, that is, $\mathrm{H}_{E}=\sum_{k} \omega_{k}\left(b_{k}^{\dagger} b_{k}+\frac{1}{2} \mathbb{1}\right)$, where $b_{k}^{\dagger}$ and $b_{k}$ are the creation and annihilation operators of the environmental mode $k$, respectively, the logarithms of the partition functions attain a simple form:

$$
\ln \left[Z\left(\beta_{i}, \mathrm{H}_{E}\right)\right]=\sum_{k}\left[-\frac{\beta_{i} \omega_{k}}{2}-\ln \left(1-e^{-\beta_{i} \omega_{k}}\right)\right],
$$

where $i=1$ and 2, which then only depends on the oscillators frequencies $\omega_{k}$ and the known inverse temperatures $\beta_{i}$.

Inequality (22) holds regardless of the choice of the actual coupling between the system and the environment. As an example of our method, in Fig. 3, we have, however, deployed a particular exactly solvable system-environment model leading to the dephasing channel of the system described in Ref. [1]. In this model, the system is a qubit coupled to environment consisting of harmonic oscillators and the total Hamiltonian, consisting of the Hamiltonians of the system $\mathrm{H}_{S}$, the environment $\mathrm{H}_{E}$, and the interaction Hamiltonian $\mathrm{H}_{I}$, governing the composite evolution can be written as

$$
\begin{aligned}
\mathrm{H} & =\mathrm{H}_{S}+\mathrm{H}_{E}+\mathrm{H}_{I} \\
& =\frac{\omega_{0}}{2} \sigma_{3}+\sum_{k} \omega_{k}\left(b_{k}^{\dagger} b_{k}+\frac{1}{2} \mathbb{1}\right)+\sum_{k} \sigma_{3} \otimes\left(g_{k} b_{k}^{\dagger}+g_{k}^{*} b_{k}\right),
\end{aligned}
$$

$g_{k}$ describes the strength at which the system couples to different modes. Fixing the initial composite system state as $\varrho_{S+E}=\varrho \otimes \xi\left(\beta_{i}\right)$, we get an analytical solution for the qubit system dynamics

$$
\varrho=\left(\begin{array}{ll}
\varrho_{00} & \varrho_{01} \\
\varrho_{10} & \varrho_{11}
\end{array}\right) \mapsto \mathcal{E}_{i}^{(t)}(\varrho)=\left(\begin{array}{cc}
\varrho_{00} & \Gamma_{i}(t) \varrho_{01} \\
\Gamma_{i}(t) \varrho_{10} & \varrho_{11}
\end{array}\right)
$$

where $\Gamma_{i}(t)=\exp \left[-\sum_{k} \frac{4\left|g_{k}\right|^{2}}{\omega_{k}^{2}} \operatorname{coth}\left(\frac{\omega_{k}}{2 T_{i}}\right)\left[1-\cos \left(\omega_{k} t\right)\right]\right]$; we refer the reader to [1] for further details of this model.

For simplicity, we will only consider environment consisting of a single oscillator; this is also the case in Fig. 3. By fixing two temperatures of the environmental initial state, $T_{1}, T_{2}$, and a single oscillator frequency $\omega$, which we want to probe, we obtain two dephasing channels $\mathcal{E}_{1}^{(t)}$ and $\mathcal{E}_{2}^{(t)}$ of the form Eq. (B3) and according to (A11) their $\alpha$ fidelity is equal to

$$
\begin{aligned}
\mathcal{F}_{\alpha}\left(\mathcal{E}_{1}^{(t)}, \mathcal{E}_{2}^{(t)}\right) & \\
= & \left(\frac{1+C_{g}(t)^{\operatorname{coth}\left(\frac{1}{2} \omega \beta_{2}\right)}}{2}\right)^{1-\alpha}\left(\frac{1+C_{g}(t)^{\operatorname{coth}\left(\frac{1}{2} \omega \beta_{1}\right)}}{2}\right)^{\alpha} \\
& +\left(\frac{1-C_{g}(t)^{\operatorname{coth}\left(\frac{1}{2} \omega \beta_{2}\right)}}{2}\right)^{1-\alpha}\left(\frac{1-C_{g}(t)^{\operatorname{coth}\left(\frac{1}{2} \omega \beta_{1}\right)}}{2}\right)^{\alpha},
\end{aligned}
$$

for $C_{g}(t)=\exp \left[-\frac{4|g|^{2}}{\omega^{2}}(1-\cos \omega t)\right]$. Importantly, the function $C_{g}(t)$ is the only term involving both the coupling strength $g$ and time $t$. It is easily verified that for any $|g| / \omega \geqslant 1 / \sqrt{8}$ the function $C_{g}(t)$ can be kept constant in value by choosing $t$ appropriately. Therefore we can conclude that any coupling (in units of $\omega$ ) stronger than $1 / \sqrt{8}$ can only make the number $\inf _{t} \mathcal{F}_{\alpha}\left(\mathcal{E}_{1}^{(t)}, \mathcal{E}_{2}^{(t)}\right)$ smaller and consequently our protocol to work better. For demonstration, we have chosen 
$|g| / \omega=1, T_{1} / \omega=0.25$, and $T_{2} / \omega=0.75$. We can therefore plot $\inf _{t} \ln \left[\mathcal{F}_{\alpha}\left(\mathcal{E}_{1}^{(t)}, \mathcal{E}_{2}^{(t)}\right)\right]$ as a function of $\alpha$, which is the right-hand side of inequality (22). Also, by using the same temperatures $T_{1}$ and $T_{2}$ and varying the frequency value in Eq. (B1), we can plot the left-hand side of inequality (22) as a function of $\alpha$ and compare it with the plot of the right-hand side. Whenever we see violation of inequality (22), we know that these frequency values cannot have led to the induced dynamics. In accordance, plots with frequencies satisfying and violating inequality (22) are presented in Fig. 3.

\section{APPENDIX C}

In application $\mathrm{C}$ (quantum thermometry), we continue on considering a system coupled to a thermal environment. This time we assume to know the oscillator frequencies of the Hamiltonian $\mathrm{H}_{E}=\sum_{k} \omega_{k}\left(b_{k}^{\dagger} b_{k}+\frac{1}{2} \mathbb{1}\right)$ and again we check the induced dynamics for two different temperatures: $T_{0}=0$ and $T$ that is unknown. Since $\xi(\infty)=\bigotimes_{k}|0\rangle\langle 0|$, the $\alpha$-Rényi divergence between the initial states of the environment in the inverse temperatures $\beta_{0}=\infty$ and $\beta=1 / k_{B} T$ can be calculated that

$$
\begin{aligned}
S_{\alpha} & \left(\xi\left(\beta_{0}\right), \xi(\beta)\right) \\
& =\frac{1}{\alpha-1} \ln \left[\operatorname{tr}\left[\left(\xi(\beta)^{\frac{1-\alpha}{2 \alpha}} \xi\left(\beta_{0}\right) \xi(\beta)^{\frac{1-\alpha}{2 \alpha}}\right)^{\alpha}\right]\right] \\
& =\frac{1-\alpha}{\alpha-1} \ln \left[e^{-\frac{1}{2} \sum_{k} \beta \hbar \omega_{k}} / Z\left(\beta, \mathrm{H}_{E}\right)\right] \\
& =\frac{1-\alpha}{\alpha-1} \ln \left[\prod_{k}\left(1-e^{-\beta \hbar \omega_{k}}\right)\right],
\end{aligned}
$$

with $\beta=1 / k_{B} T$ and the last equality follows from inserting $Z\left(\beta, \mathrm{H}_{E}\right)$ from Eq. (B1). Similarly, we confirm that

$$
S_{\alpha}\left(\xi(\beta), \xi\left(\beta_{0}\right)\right)=\frac{\alpha}{\alpha-1} \ln \left[\prod_{k}\left(1-e^{-\beta \omega_{k}}\right)\right] .
$$

Consequently, from inequality (5), one confirms that

$$
\begin{aligned}
& \prod_{k}\left(1-e^{-\hbar \omega_{k} / k_{B} T}\right) \\
& \quad \leqslant \inf _{t \geqslant 0}\left\{\begin{array}{ll}
\mathcal{F}_{\alpha}\left(\mathcal{E}_{T}^{(t)}, \mathcal{E}_{0}^{(t)}\right)^{\frac{1}{\alpha}}, & \text { for } \alpha \in\left(0, \frac{1}{2}\right) \\
\mathcal{F}_{\alpha}\left(\mathcal{E}_{0}^{(t)}, \mathcal{E}_{T}^{(t)}\right)^{\frac{1}{1-\alpha}}, & \text { for } \alpha \in\left[\frac{1}{2}, 1\right)
\end{array},\right.
\end{aligned}
$$

where $\mathcal{E}_{0}^{(t)}$ and $\mathcal{E}_{T}^{(t)}$ are the dynamics induced by $\beta_{0}$ and $\beta_{T}$, respectively. From this inequality the temperature $T$ can be numerically estimated. For simplicity, we again consider the case of only a single harmonic oscillator mode. In this case, the above relation can be solved in terms of the temperature $T$ analytically: the solution is presented in inequality (24).

Notice, that the above inequality (C3) holds modelindependently even without a priori knowledge of the coupling between the system and the environment. As a concrete example, in Fig. 4, we have demonstrated the power of our method by considering the Jaynes-Cummings model of a qubit coupled to a thermal environment consisting of a single harmonic oscillator. In such a model, the total Hamiltonian of the system-environment composite reads

$$
\begin{aligned}
\mathrm{H} & =\mathrm{H}_{S}+\mathrm{H}_{E}+\mathrm{H}_{I} \\
& =\omega_{0} \sigma_{+} \sigma_{-}+\omega\left(b^{\dagger} b+\frac{1}{2} \mathbb{1}\right)+g\left(\sigma_{+} \otimes b+\sigma_{-} \otimes b^{\dagger}\right),
\end{aligned}
$$

where $\sigma_{+}=|1\rangle\langle 0|$ and $\sigma_{-}=|0\rangle\langle 1|$ are the raising and lowering operators of the qubit system, respectively, and $g$ describes the coupling strength. Assume that an initial separable state of the composite system $\varrho \otimes \xi(\beta)$ evolves according to this Hamiltonian. Then, in the resonant case of matching system and environment frequencies $\Delta=\omega-\omega_{0}=0$, the reduced dynamics take a relatively simple form

$$
\varrho=\left(\begin{array}{ll}
\varrho_{00} & \varrho_{01} \\
\varrho_{10} & \varrho_{11}
\end{array}\right) \mapsto \mathcal{E}_{T}^{(t)}(\varrho)=\left(\begin{array}{lc}
a_{T}(t) \varrho_{00}+\left[1-b_{T}(t)\right] \varrho_{11} & c_{T}(t)^{*} \varrho_{01} \\
c_{T}(t) \varrho_{10} & {\left[1-a_{T}(t)\right] \varrho_{00}+b_{T}(t) \varrho_{11}}
\end{array}\right)
$$

where the coefficients

$$
\begin{aligned}
& a_{T}(t)=\operatorname{tr}\left[\mathrm{C}^{\dagger}(\hat{n}, t) \mathrm{C}(\hat{n}, t) \xi(\beta)\right], \\
& b_{T}(t)=\operatorname{tr}\left[\mathrm{C}^{\dagger}(\hat{n}+1, t) \mathrm{C}(\hat{n}+1, t) \xi(\beta)\right], \\
& c_{T}(t)=\operatorname{tr}[\mathrm{C}(\hat{n}+1, t) \mathrm{C}(\hat{n}, t) \xi(\beta)]
\end{aligned}
$$

depend on the operator $\mathrm{C}(\hat{n}, t)=\cos (|g| t \sqrt{\hat{n}})$, $\hat{n}=b^{\dagger} b \quad$ [38]. Inserting the thermal state $\xi(\beta)$ in the above formulas we get, e.g., $a_{T}(t)=$ $\sum_{n=0}^{N} e^{-\beta \hbar \omega(n+1 / 2)} \cos ^{2}(|g| t \sqrt{n}) / \sum_{n=0}^{\infty} e^{-\beta \hbar \omega(n+1 / 2)}$.

Although in principle $N \rightarrow \infty$, in lack of a closed form solution, we have truncated the series to $N=10$ (similarly for $b_{T}(t)$ and $\left.c_{T}(t)\right)$. The error due to this estimation is negligible because for large $N$ the dominating exponent factor makes the summand minuscule.

Our numerics suggest that, even though the induced dynamics are not purely dephasing, the choice $\varrho_{1}=\varrho_{2}=$ $|+\rangle\langle+|$ minimizes the channel $\alpha$ fidelity $\inf _{t \geqslant 0} \mathcal{F}_{\alpha}\left(\mathcal{E}_{0}^{(t)}, \mathcal{E}_{T}^{(t)}\right)$ also in this case. Therefore the temperature can, in fact, be directly estimated in terms of the Kullback-Leibler divergence $S_{1}\left(\mathcal{E}_{0}(\varrho)|| \mathcal{E}_{T}(\varrho)\right), \varrho=|+\rangle\langle+|$, as shown in inequalities (27) and (28) and which we have plotted in Fig. 4. The remaining optimization with respect to time $t$ has been done numerically by using the build in algorithms of MATHEMATICA. Finally, we note that, because of the relation of the coupling strength $g$ and $t$ in the terms $\mathrm{C}(\hat{n}, t)$ defining the dynamics and the time optimization involved in our protocol, the upper and lower bounds presented in Fig. 4 are independent of the strength of the coupling as long as it is nonvanishing. 
[1] H. P. Breuer and F. Petruccione, The Theory of Open Quantum Systems (Oxford University Press, New York, 2007), Vol. 1, p. 227-228.

[2] A. Recati, P. O. Fedichev, W. Zwerger, J. von Delft, and P. Zoller, Phys. Rev. Lett. 94, 040404 (2005).

[3] M. Bruderer and D. Jaksch, New J. Phys. 8, 87 (2006).

[4] T. H. Johnson, S. R. Clark, M. Bruderer, and D. Jaksch, Phys. Rev. A 84, 023617 (2011).

[5] R. Dorner, S. R. Clark, L. Heaney, R. Fazio, J. Goold, and V. Vedral, Phys. Rev. Lett. 110, 230601 (2013).

[6] S. McEndoo, P. Haikka, G. De Chiara, G. M. Palma, and S. Maniscalco, Europhys. Lett. 101, 60005 (2013).

[7] P. Haikka, S. McEndoo, and S. Maniscalco, Phys. Rev. A 87, 012127 (2013).

[8] P. Haikka and K. Mølmer, Phys. Rev. A 89, 052114 (2014).

[9] V. Jagadish and A. Shaji, Ann. Phys. 362, 287 (2015).

[10] S. Manikandan, V. Jagadish, and A. Shaji, arXiv:1507.00583

[11] M. A. Nielsen and I. L. Chuang, Phys. Rev. Lett. 79, 321 (1997).

[12] M. Hillery, M. Ziman, and V. Bužek, Phys. Rev. A 66, 042302 (2002).

[13] M. Hillery, M. Ziman, and V. Bužek, Phys. Rev. A 73, 022345 (2006).

[14] T. Heinosaari and M. Tukiainen, Quant. Info. Proc. 14, 3097 (2015).

[15] G. Vidal and J. I. Cirac, arXiv:quant-ph/0012067v1.

[16] G. Vidal, L. Masanes, and J. I. Cirac, Phys. Rev. Lett. 88, 047905 (2002).

[17] M. Hillery, V. Bužek, and M. Ziman, Phys. Rev. A 65, 022301 (2002).

[18] M. Hillery, M. Ziman, and V. Bužek, Phys. Rev. A 69, 042311 (2004).

[19] A. Rényi, On Measures of Information and Entropy, in Proceedings of the Fourth Berkeley Symposium on Mathematical Statistics and Probability, Vol. 1: Contributions to the Theory of Statistics (University of California Press, Oakland, 1961), pp. 547-561.

[20] M. Müller-Lennert, F. Dupuis, O. Szehr, S. Fehr, and M. Tomamichel, J. Math. Phys. 54, 122203 (2013).

[21] M. Wilde, A. Winter, and D. Yang, Commun. Math. Phys. 331, 593 (2014).

[22] S. Beigi, J. Math. Phys. 54, 122202 (2013).

[23] N. Datta and F. Leditzky, J. Phys. A: Math. Theor. 46, 045304 (2014).

[24] K. Audenaert and N. Datta, J. Math. Phys. 56, 022202 (2015).
[25] E. Carlen, R. Frank, and E. Lieb, Linear Algebra Its Appl. 490, 174 (2016).

[26] M. Raginsky, Phys. Lett. A 290, 11 (2001).

[27] V. Belavkin, G. D'Ariano, and M. Raginsky, J. Math. Phys. 46, 062106 (2005).

[28] M. Ziman, Phys. Rev. A 77, 062112 (2008).

[29] M. Ziman and M. Sedlák, J. Mod. Opt. 57, 253 (2010).

[30] A. Gilchrist, N. K. Langford, and M. A. Nielsen, Phys. Rev. A 71, 062310 (2005).

[31] M. Nielsen and I. Chuang, Quantum Computation and Quantum Information, 10th ed. (Cambridge University Press, New York, 2010).

[32] M. Mosonyi, arXiv:1310.7525v3.

[33] V. Bužek, M. Hillery, M. Ziman, and M. Roško, Quantum Inf. Proc. 5, 313 (2006).

[34] M. B. Ruskai, S. Szarek, and E. Werner, Lin. Alg. Appl. 347, 159 (2002).

[35] J. Miszczak et al., Quantum Inf. Comput. 9, 0103 (2009).

[36] E. Lieb and W. Thirring, Studies in Mathematical Physics (Princeton University Press, Princeton, 1976).

[37] H. Araki, Lett. Math. Phys. 19, 167 (1990).

[38] A. Smirne and B. Vacchini, Phys. Rev. A 82, 022110 (2010).

[39] S. Jevtic, D. Newman, T. Rudolph, and T. M. Stace, Phys. Rev. A 91, 012331 (2015).

[40] M. Brunelli, S. Olivares, M. Paternostro, and M. G. A. Paris, Phys. Rev. A 86, 012125 (2012).

[41] K. D. B. Higgins, B. W. Lovett, and E. M. Gauger, Phys. Rev. B 88, 155409 (2013).

[42] T. H. Johnson, F. Cosco, M. T. Mitchison, D. Jaksch, and S. R. Clark, Phys. Rev. A 93, 053619 (2016).

[43] L. A. Correa, M. Mehboudi, G. Adesso, and A. Sanpera, Phys. Rev. Lett. 114, 220405 (2015).

[44] Á. Rivas, S. Huelga, and M. Plenio, Rev. Prog. Phys. 77, 094001 (2014).

[45] H.-P. Breuer, E.-M. Laine, J. Piilo, and B. Vacchini, Rev. Mod. Phys. 88, 021002 (2016).

[46] M. Hinarejos, M.-C. Bañuls, A. Pérez, and I. de Vega, arXiv:1606.01185v1.

[47] H. T. Quan, Z. Song, X. F. Liu, P. Zanardi, and C. P. Sun, Phys. Rev. Lett. 96, 140604 (2006).

[48] P. Haikka, J. Goold, S. McEndoo, F. Plastina, and S. Maniscalco, Phys. Rev. A 85, 060101(R) (2012). 